ARTICLE

Received 22 Dec 2015 | Accepted 6 Sep 2016 | Published 18 Oct $2016 \quad$ DOl: 10.1038/ncomms13132 OPEN

\title{
Engineering prokaryotic channels for control of mammalian tissue excitability
}

\author{
Hung X. Nguyen ${ }^{1}$, Robert D. Kirkton ${ }^{1} \&$ Nenad Bursac ${ }^{1}$
}

The ability to directly enhance electrical excitability of human cells is hampered by the lack of methods to efficiently overexpress large mammalian voltage-gated sodium channels (VGSC). Here we describe the use of small prokaryotic sodium channels $\left(\mathrm{BacNa}_{\mathrm{v}}\right.$ ) to create de novo excitable human tissues and augment impaired action potential conduction in vitro. Lentiviral co-expression of specific $\mathrm{BacNa}_{\mathrm{v}}$ orthologues, an inward-rectifying potassium channel, and connexin-43 in primary human fibroblasts from the heart, skin or brain yields actively conducting cells with customizable electrophysiological phenotypes. Engineered fibroblasts ('E-Fibs') retain stable functional properties following extensive subculture or differentiation into myofibroblasts and rescue conduction slowing in an in vitro model of cardiac interstitial fibrosis. Co-expression of engineered $\mathrm{BaCNa}_{v}$ with endogenous mammalian VGSCs enhances action potential conduction and prevents conduction failure during depolarization by elevated extracellular $\mathrm{K}^{+}$, decoupling or ischaemia. These studies establish the utility of engineered $\mathrm{BacNa}_{v}$ channels for induction, control and recovery of mammalian tissue excitability.

\footnotetext{
${ }^{1}$ Department of Biomedical Engineering, Duke University, 101 Science Drive, Room 1427, Fitzpatrick CIEMAS, Durham, North Carolina 27708, USA. Correspondence and requests for materials should be addressed to N.B. (email: nbursac@duke.edu).
} 
$\mathrm{V}$ oltage-gated sodium channels (VGSCs) exist in all animal organisms and govern processes fundamental to life including neuronal communication, muscle contractions or hormonal release ${ }^{1}$. In electrically excitable tissues, VGSCs enable firing and spread of action potentials (APs) and their lossof-function mutations ${ }^{2}$ cause a variety of neuronal ${ }^{3-6}$, cardiac $^{7,8}$ and skeletal muscle ${ }^{9,10}$ disorders. Similarly, acute tissue injuries resulting in permanent excitability loss (for example, stroke, spinal cord injury and heart attack) are associated with long-term disability and death. In addition to excitable tissues, the VGSCs are expressed in many unexcitable cells including macrophages, microglia and islet $\beta$-cells, where they play important roles in phagocytosis, migration, insulin secretion and other cellular processes ${ }^{11}$. The ion-conducting $\alpha$-subunits of mammalian VGSCs have four structurally homologous domains, each consisting of $\sim 300$ amino acids ${ }^{12}$. These four domains are simultaneously transcribed and translated from a single, relatively large $(>6 \mathrm{~kb})$ gene. This large gene size precludes stable and efficient expression of mammalian VGSCs in primary cells, a strategy that could potentially lead to new remedies for impaired tissue excitability.

The recent discovery of a large bacterial VGSC family $\left(\mathrm{BacNa}_{\mathrm{v}}\right)$ has provided novel insights into the structure and biophysical properties of eukaryotic $\mathrm{VGSCs}^{13-17}$. In contrast to their eukaryotic counterparts, $\mathrm{BacNa}_{\mathrm{v}}{ }^{13}$ consist of four identical domains, individually transcribed and translated from single genes of only $\sim 900 \mathrm{bp}$ in size. Although the small size of $\mathrm{BaCNa}_{\mathrm{v}}$ facilitates their overexpression and purification for crystallography studies ${ }^{18}$, it is plausible that $B a c N a_{v}$ genes could be efficiently packaged into viral vectors, either alone or with other ion channel genes, and used to stably introduce or modify electrical excitability of mammalian tissues. Furthermore, utilization of $\mathrm{BacNa}_{\mathrm{v}}$ orthologues with diverse biophysical properties $^{13-16,19,20}$, combined with targeted channel mutations, may allow custom engineering of cellular electrophysiological phenotypes tailored to specific tissue types, diseases and individuals.

Here we combined electrophysiological, computational and genetic engineering techniques to establish a versatile $\mathrm{BacNa}_{\mathrm{v}}{ }^{-}$ based platform that enables stable conversion of unexcitable primary human fibroblasts into AP conducting cells that can successfully recover conduction slowing in an in vitro model of cardiac interstitial fibrosis. Moreover, we demonstrate that direct expression of engineered $\mathrm{BacNa}_{\mathrm{v}}$ channels in mammalian excitable cells can enhance their excitability and prevent loss of AP conduction in pathological conditions when endogenous $\mathrm{Na}^{+}$channels fail to activate.

\section{Results}

Genetic engineering of actively conducting human fibroblasts. To stably convert unexcitable primary fibroblasts into actively conducting cells (named engineered fibroblasts or 'E-Fibs'), we first explored a $\mathrm{BacNa}_{\mathrm{v}}$ variant from Roseobacter denitrificans, $\mathrm{Na}_{\mathrm{v}}$ RosD G217A, shown in our preliminary tests to be capable of generating AP propagation when transfected in a monolayer of HEK293 cells stably expressing Kir2.1 and connexin-43 (Cx43). We generated a high-titre bi-cistronic lentivirus pRRL-CMVRosDG217A-P2A-eGFP-Kir2.1 encoding both $\mathrm{Na}_{\mathrm{v}} \mathrm{RosD}$ G217A and inward-rectifier potassium channel Kir2.1 (gene KCNJ2) fused with enhanced green fluorescent protein (eGFP) (eGFP-Kir2.1), separated by the self-cleaving peptide P2A. Adult human dermal fibroblasts (HDFs) transduced with this virus stably expressed inward-rectifier $\mathrm{K}^{+}$current, $I_{\mathrm{K} 1}$ (Fig. 1a,b), and bacterial $\mathrm{Na}^{+}$current, $I_{\mathrm{NaRosD}}$ G217A $($ Fig. 1c,d), at much higher levels compared with endogenous currents, and exhibited a significantly hyperpolarized resting membrane potential of $-78.4 \pm 0.2 \mathrm{mV}(n=6)$ compared with that of wild-type (wt)-HDFs $(-23.3 \pm 2.9 \mathrm{mV} ; n=5)$. The resulting voltage dependencies of activation and inactivation of $\mathrm{Na}_{\mathrm{v}} \mathrm{Ros} \mathrm{D}$ G217A (Fig. 1e) in transduced HDFs were similar to those previously reported in transfected HEK293s (ref. 15). Importantly, transduced HDFs became electrically excitable, whereby injection of $1 \mathrm{~ms}$ current pulses of increasing amplitude elicited reproducible firing of an 'all-or-none' AP (Fig. 1f). These results demonstrated the ability of $\mathrm{BaCNa}_{\mathrm{v}}$ to yield creation of de novo electrically excitable cells.

To enable electrical coupling in excitable HDFs, we co-transduced them with a pRRL-CMV-Cx43-P2A-mCherry lentivirus. Transduced cells robustly expressed membrane-bound Cx43 gap junctions (Fig. 1g), which resulted in improved intercellular coupling compared with wt-HDFs as revealed by fluorescence recovery after photobleaching ${ }^{21}$, albeit not to the same level as measured in neonatal rat cardiomyocytes (Fig. 1h). These engineered HDFs (E-HDFs) were then cultured at confluence in $2 \mathrm{~cm}$ diameter monolayers in a random or aligned configuration (Supplementary Fig. 1). When electrically stimulated by a bipolar platinum electrode, randomly oriented E-HDFs supported circular AP spread at an average conduction velocity (CV) of $3.63 \pm 0.23 \mathrm{~cm} \mathrm{~s}^{-1}$ (Fig. 1i and Supplementary Video 1). As expected, aligned E-HDFs exhibited elliptical (anisotropic) AP spread with higher CVs along $\left(3.86 \pm 0.14 \mathrm{~cm} \mathrm{~s}^{-1}, n=5\right)$ versus across $\left(2.07 \pm 0.19 \mathrm{~cm} \mathrm{~s}^{-1}\right.$, $n=5$ ) cell orientation (Fig. $1 \mathrm{j}$ and Supplementary Video 1). Although the above approach enabled AP propagation in initially unexcitable human fibroblasts, CVs were low, which could be at least in part attributed to the relatively slow activation kinetics of $\mathrm{Na}_{\mathrm{v}}$ RosD G217A.

Improved E-Fib electrical properties with NavSheP mutants. To augment the excitability and conduction of E-HDFs, we developed a combined computational and experimental strategy to screen and identify $\mathrm{BacNa}_{\mathrm{v}}$ variants with more favourable properties (Fig. 2a). We set to explore the $\mathrm{BacNa}_{\mathrm{v}}$ orthologue from Shewanella putrefaciens, $\mathrm{Na}_{\mathrm{v}} \mathrm{SheP}$, for its fast current kinetics ${ }^{15}$. As the wt $\mathrm{Na}_{\mathrm{v}} \mathrm{SheP}$ channel has a highly hyperpolarized voltage dependency of steady-state inactivation, it does not yield AP firing when co-expressed with Kir2.1. We thus proceeded to shift the activation and inactivation curves of $\mathrm{Na}_{v} \mathrm{SheP}$ to more depolarized voltages based on the previous studies of specific residues in $\mathrm{NaChBac}$ channels ${ }^{22}$ and significant homology between different $\mathrm{BacNa}_{\mathrm{v}}$ orthologues (Supplementary Fig. 2); we created a library of $\mathrm{Na}_{\mathrm{v}} \mathrm{SheP}$ mutants where either the D60 or E43 residue was substituted with other standard amino acids. These $38 \mathrm{Na}_{\mathrm{v}}$ SheP mutants were transiently expressed in a monoclonal HEK293 line stably expressing Kir2.1 and the resulting sodium current was recorded by voltage clamp. The E43 mutants exhibited highly depolarized voltage dependence of activation (Supplementary Table 1); therefore, we focused on detailed characterization of D60 mutants. The D60 mutant channels exhibited a wide range of steady-state and kinetic parameters (Supplementary Fig. 3 and Supplementary Table 1) and predominantly exhibited fast kinetics compared with the $\mathrm{Na}_{\mathrm{v}}$ RosD channel (Supplementary Fig. 3c,d and Supplementary Table 1). Based on the recorded parameters, we applied computational modelling to infer AP upstroke, threshold, duration and CV that would result from the same expression level of different $\mathrm{Na}_{\mathrm{v}}$ SheP mutants in an electrically coupled, Kir2.1 HEK293 line (Fig. 2b-e and Supplementary Fig. 4) and constructed $\mathrm{Na}_{\mathrm{v}}$ SheP D60X-Kir2.1 lentiviruses for three D60X mutants (D60A, D60N and D60S) that exhibited the steepest AP 
a

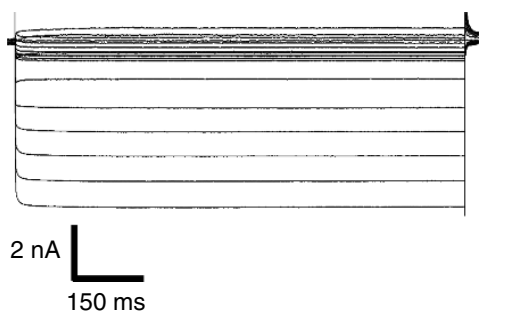

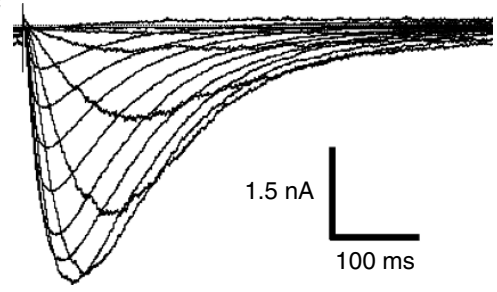

e

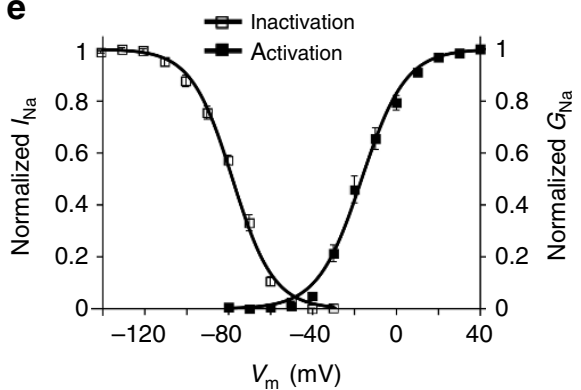

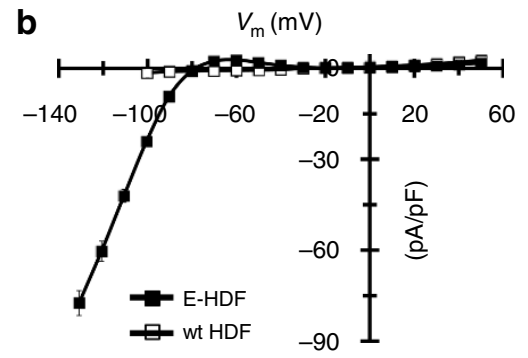

b d
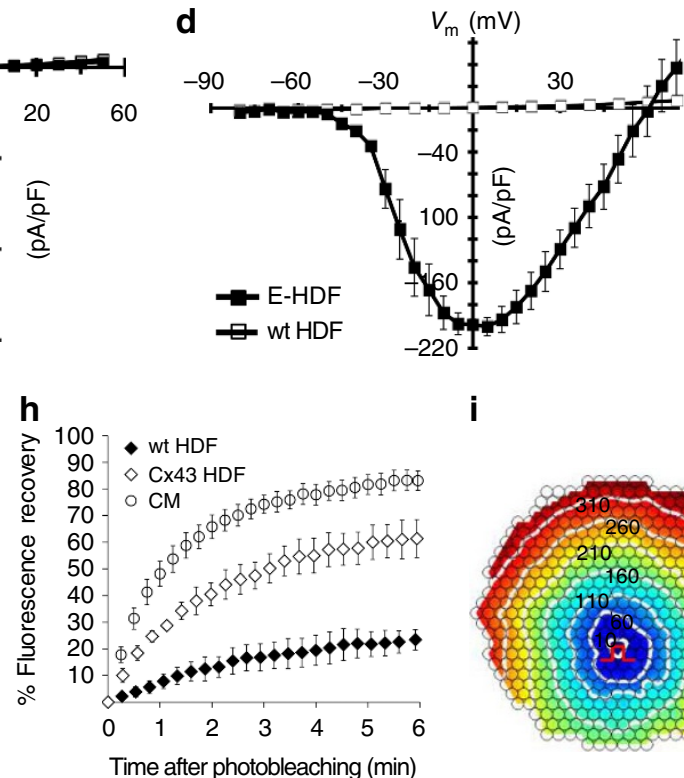

i

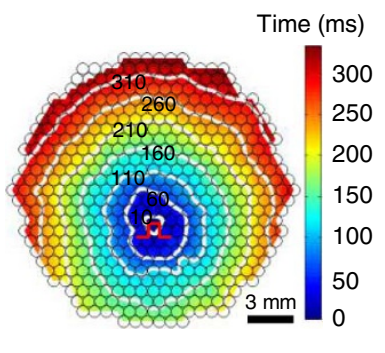

f

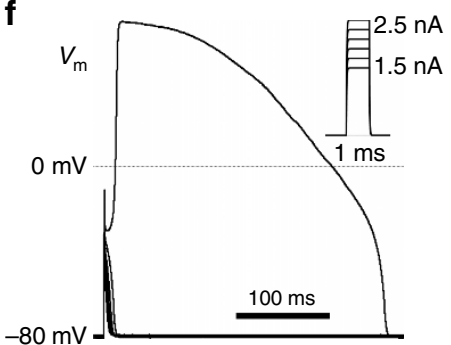

j

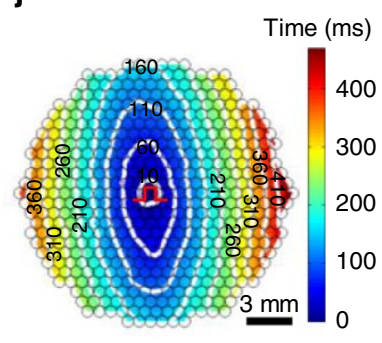

Figure 1 | Generation of actively conducting human E-Fibs. (a) Voltage-clamp recording of $I_{K 1}$ in E-HDFs. (b) Steady-state $I_{K 1}-V$ curves in E-HDF and wt (unexcitable) HDF. (c-e) Recording of $I_{\mathrm{Na}}(\mathbf{c})$, peak $I_{\mathrm{Na}}-\mathrm{V}$ curve (d) and voltage dependence of $I_{\mathrm{Na}}$ activation and steady-state inactivation (e) in E-HDFs expressing $\mathrm{Na}_{\mathrm{v}}$ RosD G217A. In b,d,e: $n=8-10$. Data in a-e recorded at $25^{\circ} \mathrm{C}$. (f) All-or-none AP response in E-HDFs induced by current pulses ( $I_{\mathrm{m}}$ ) of increasing amplitude. ( $\mathbf{g}, \mathbf{h})$ Stable overexpression of $\mathrm{Cx} 43$ in E-HDFs $(\mathbf{g})$ results in formation of functional gap junctions, shown by increased recovery of fluorescence after photobleaching, albeit at a slower rate than that of neonatal rat cardiomyocytes $(\mathrm{CM})(\mathbf{h}, n=6)$. (i,j) Representative isochrone maps of AP conduction in electrically stimulated isotropic (i) and anisotropic (j) monolayers of E-HDFs stably co-expressing Kir2.1, NavesosD G217A and Cx43. Pulse signs indicate position of stimulating electrode. Circles denote 504 recording sites. All electrophysiological data obtained at $37^{\circ} \mathrm{C}$, unless otherwise specified. Error bars indicate s.e.m.

upstrokes and highest CVs among different $\mathrm{BacNa}_{\mathrm{v}}$ models. All three mutants exhibited significantly faster kinetics compared with $\mathrm{Na}_{\mathrm{v}}$ RosD G217A in transduced cells (Fig. 2f,g), which allowed generation of APs with shorter duration and sharper upstroke velocity (Fig. $2 \mathrm{~h}-\mathrm{j}$ ). Co-transduction of these viruses with $\mathrm{Cx} 43$ in $\mathrm{HDF}$ yielded a 2.5 -fold improvement in $\mathrm{CV}$ compared with the use of $\mathrm{Na}_{\mathrm{v}}$ RosD (Fig. 2k,l and Supplementary Video 2). This demonstrated the potential for combining $\mathrm{BacNa}_{\mathrm{v}}$ mutagenesis and computational modelling, to engineer primary human fibroblasts with desired electrophysiological phenotypes.

Fine-tuning of E-Fib electrical properties via FACS. The use of fluorescence reporters in multi-cistronic lentiviruses (for example, pRRL-CMV-SheP D60N-P2A-eGFP-Kir2.1) allowed us to further vary $I_{\mathrm{NaSheP}}$ and $I_{\mathrm{K} 1}$ levels, and characterize their functional effects on E-HDFs. Using fluorescence-activated cell sorting (FACS), we sorted E-HDFs into three populations with low, medium and high GFP expression levels (Supplementary Fig. 5a), which yielded corresponding low, medium and high levels of $I_{\mathrm{K} 1}$ (Fig. 3a) and $I_{\text {NaSheP }}$ (Fig. 3b), and distinct changes in AP shape (Fig. 3c). Specifically, a trend towards more hyperpolarized resting membrane potential (Fig. 3d, one-way analysis of variance, $P=0.15$ ) and the significantly higher maximum upstroke velocity (Fig. $3 \mathrm{e}$ ) could be attributed to higher $I_{\mathrm{K} 1}$ and $I_{\mathrm{NaSheP}}$, respectively, whereas the increased APD (Fig. 3f) resulted from net depolarizing effect of increased $I_{\mathrm{K} 1}$ and $I_{\mathrm{NaSheP}}$ on membrane repolarization. Although higher AP upstroke velocities expectedly yielded higher CVs (Fig. 3g,h), this effect was moderate, which we hypothesized was due to simultaneous increase in $I_{\mathrm{K} 1}$ along with $I_{\mathrm{NaSheP}}$. We thus modelled concurrent increase in $I_{\mathrm{K} 1}$ and $I_{\mathrm{NaSheP}}$ to simulate the experiment (model 1; Supplementary Fig. 6) and compared resulting CVs with those caused by an increase in $I_{\mathrm{NaSheP}}$ alone (model 2; Supplementary Fig. 6). Despite the same AP upstroke velocities in both models (Supplementary Fig. 6a), CV in model 1 increased less (Supplementary Fig. 6b), because more depolarizing current was needed to overcome high $I_{\mathrm{K} 1}$, reach threshold and elicit AP (Supplementary Fig. 6c). Examining a wide range of channel conductances $\left(\overline{\mathrm{G}}_{\mathrm{Na}}\right.$ and $\left.\overline{\mathrm{G}}_{\mathrm{K} 1}\right)$ further revealed that although threshold current required to elicit AP is primarily sensitive to level of $I_{\mathrm{K} 1}$ (Supplementary Fig. 6d), AP upstroke velocity is mainly dependent on $I_{\mathrm{NaSheP}}$ (Supplementary Fig. 6e), with these two currents inducing opposite effects on CV (Supplementary 

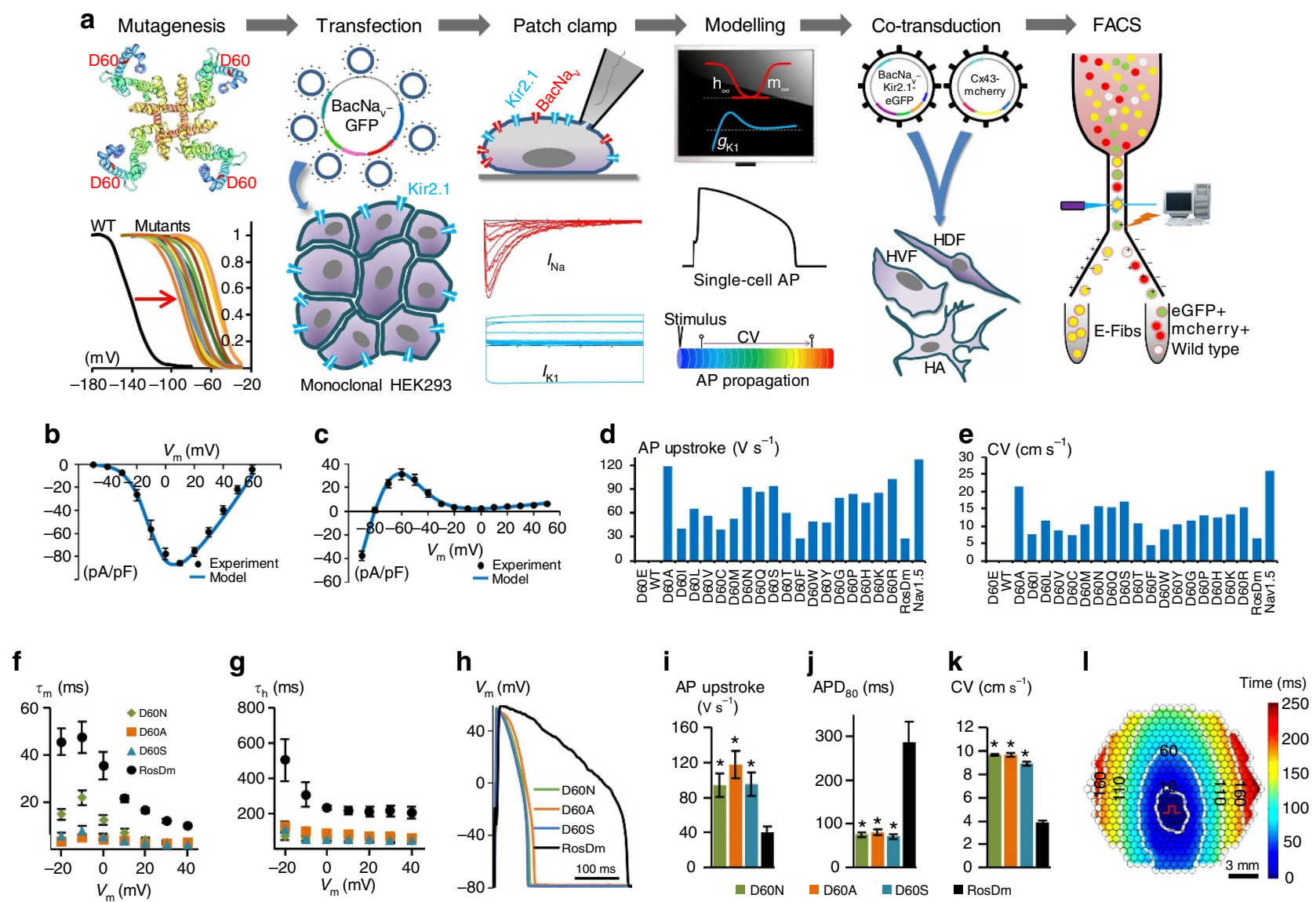

Figure 2 | Customization of E-Fib phenotype via mutagenesis. (a) BacNav-based strategy for E-Fib customization. (b) Experimental ( $n=8)$ and modelling peak $I_{\mathrm{Na}}-V$ curves. Cell membrane was held at $-80 \mathrm{mV}$ before stepping the voltage from -50 to $60 \mathrm{mV}$ in $10 \mathrm{mV}$ increments. (c) Experimental ( $\left.n=21\right)$ and modelling steady-state $I_{K}-V$ curves. Cell membrane was held at $-40 \mathrm{mV}$ before stepping the voltage from -90 to $50 \mathrm{mV}$ in $10 \mathrm{mV}$ increments. (d,e) AP initiation and conduction characteristics of $\mathrm{BaCNa}_{\mathrm{v}}$ mutant expressing E-HDFs obtained by computational modeling: upstroke velocity (d) and CV (e). For modelling, parameters derived from voltage-clamp measurements at $25^{\circ} \mathrm{C}$ were scaled for the temperature of $37^{\circ} \mathrm{C}$. Highly hyperpolarized inactivation of WT and D60E channels prevented AP initiation. $(\mathbf{f}, \mathbf{g})$ Time constants of activation $(\mathbf{f})$ and inactivation $(\mathbf{g})$ of selected Nav SheP D60X mutants compared with $\mathrm{Na}_{\mathrm{v}}$ RosD G217A (RosDm), recorded in E-HEK293 cells at $25^{\circ} \mathrm{C}(n=5)$. (h-j) When co-expressed with Kir2.1 in E-HEK293 cells, $\mathrm{Na}_{\mathrm{v}}$ SheP mutants give rise to APs (h) with faster upstroke $(\mathbf{i} ; n=10-18)$ and shorter duration $(\mathbf{j} ; n=10-18)$ than NavesD G217A ( $n=11$ for $\left.\mathbf{i}, \mathbf{j}\right)$.

(k) $\mathrm{Na}_{\mathrm{v}}$ SheP D60X expressions in anisotropic E-HDF monolayers yield faster CV than RosDm expression $(n=5-10)$. (I) Representative isochrone map of AP conduction in an electrically stimulated anisotropic monolayer of E-HDFs stably co-expressing Kir2.1, Na $\mathrm{S}_{\mathrm{S}} \mathrm{P}$ D60N and Cx43 (compare also with Fig. 1j). ${ }^{\star} P<0.001$ versus RosDm (i-k). All electrophysiological data obtained at $37^{\circ} \mathrm{C}$, unless otherwise specified. Error bars indicate s.e.m; statistical significance was determined by one-way analysis of variance, followed by Tukey's post-hoc test to calculate $P$-values.

Fig. 6f). Furthermore, as E-HDFs were also transduced with pRRL-CMV-Cx43-P2A-mCherry lentivirus, sorting cells based on mCherry fluorescence level (Supplementary Fig. 5c) allowed us to vary $\mathrm{Cx} 43$ expression and gap junctional coupling of engineered cells. Optical mapping revealed that E-HDFs with higher mCherry/Cx43 expression supported faster AP propagation (Fig. 3i), whereas contrary to eGFP sorting (Supplementary Fig. 5b), mCherry sorting had no effect on APD 80 (Supplementary Fig. 5d). Simulation results further revealed that intercellular coupling strength does not influence the relative effects of varying $I_{\mathrm{K} 1}$ and $I_{\mathrm{NaSheP}}$ on CV (Supplementary Fig. $6 \mathrm{~g}$ ).

Robustness of E-Fib electrical phenotype. To test whether E-HDFs can maintain stable electrophysiological phenotype under various perturbations, we expanded them 500 -fold over a 4 -week period, either directly or after 2 freeze-thaw cycles. In both cases, expanded E-HDFs retained proliferative potential (Fig. 4a) and were able to undergo additional rounds of expansion (for example, 100-fold, 4 passages; Fig. 4b). In addition, as fibroblasts can differentiate into smooth muscle actin expressing myofibroblasts during wound healing and tissue repair ${ }^{23}$, we treated the expanded E-HDFs with $10 \mathrm{ng} \mathrm{ml}^{-1}$ transforming growth factor- $\beta 1$ for 7 days, to induce their conversion to myofibroblasts (Fig. 4c,d). Subsequent electrophysiological analysis revealed that despite significant cell expansion or myofibroblast differentiation, the $I_{\mathrm{K} 1}, I_{\mathrm{NaSheP}}$ (Fig. 4e,f) and AP characteristics (Fig. 4g-i) of E-HDFs remained unchanged, demonstrating the stability of the acquired functional phenotype. The ability of these cells to undergo repeated freeze-thaw cycles without phenotypic alterations suggested the potential for off-the-shelf delivery applications. Electrophysiological phenotype of E-HDFs remained stable until the cells reached senescence (after additional eight passages and $\sim 10^{6}$-fold expansion; Supplementary Fig. 7a-d). Furthermore, using a classical heterologous expression system-monoclonal HEK293 line expressing Cx43, $\mathrm{K}_{\mathrm{ir}} 2.1$ and $\mathrm{Na}_{\mathrm{v}}$ SheP D60A-stable electrical properties were maintained beyond 20 passages 

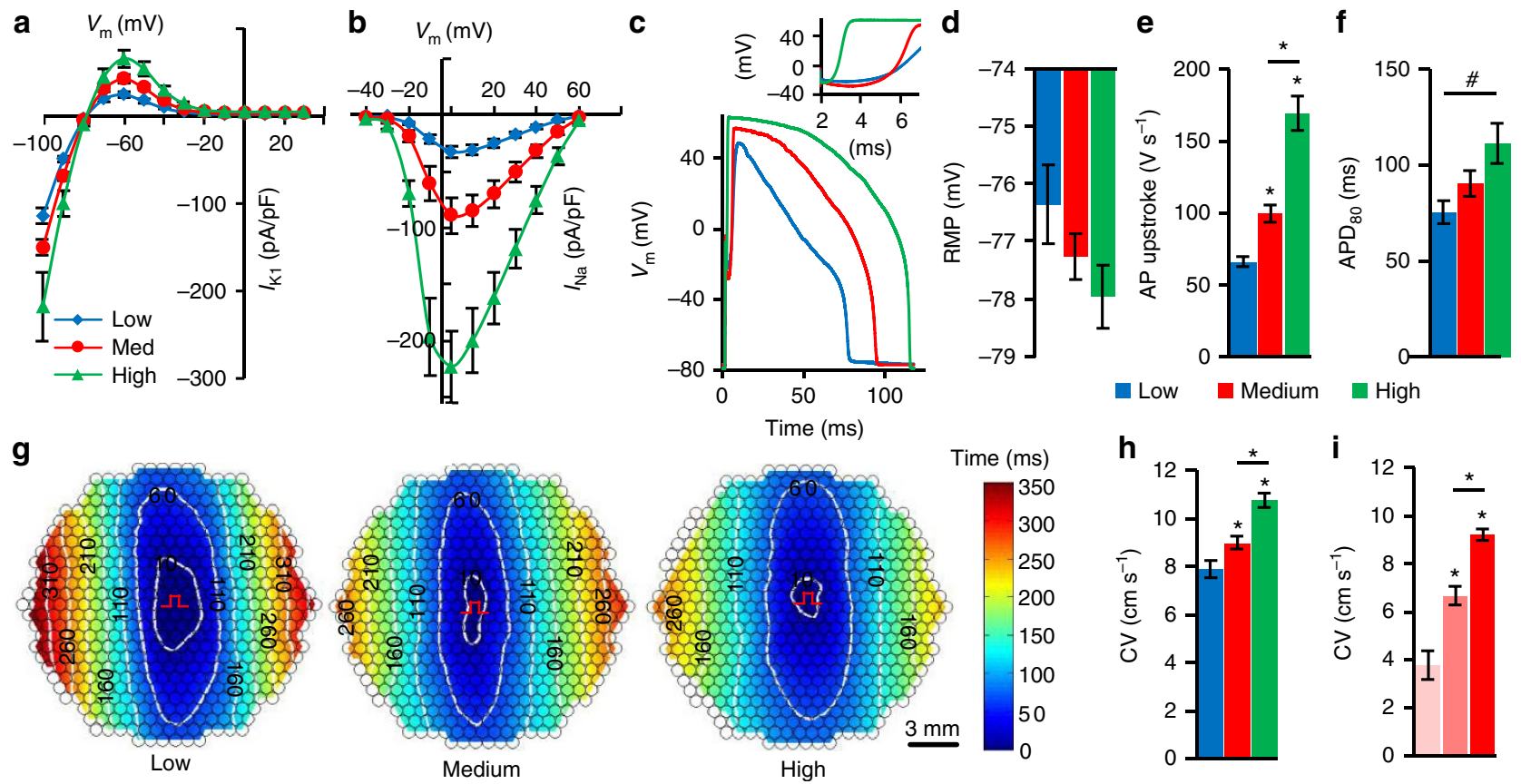

Figure 3 | Tuning electrical properties of E-Fibs via FACS. (a,b) Steady-state $I_{\mathrm{K} 1}-V$ curves $(\mathbf{a} ; n=8-12)$ and peak $I_{\mathrm{Na}}-V$ curve $(\mathbf{b} ; n=8-12)$ in three groups (blue, red and green denoting low, medium and high eGFP-expressing cells, respectively), measured at $25^{\circ} \mathrm{C}$. (c) Representative AP traces with inset showing AP upstrokes in the three eGFP-sorted E-HDF groups. (d-f) Resting membrane potential (RMP, d), maximum AP upstroke velocity (e) and APD 80 (f) in the three eGFP-sorted E-HDF groups $(n=14-18)$. (g,h) Representative isochrone maps of AP conduction in electrically stimulated anisotropic monolayers of E-HDFs $(\mathbf{g})$ showing that cells with higher eGFP expression had faster CV $(\mathbf{h} ; n=6-10)$. All E-HDFs were first sorted for high Cx43-mCherry level to ensure strong intercellular coupling. Pulse signs indicate location of stimulating electrode. Circles denote 504 recording sites. (i) CV in electrically stimulated monolayers of three mCherry-sorted E-HDF groups (left, middle and right bars denoting low, medium and high mCherry-expressing cells, respectively; $n=5)$. $\# P<0.01$ in $\mathbf{f} ;{ }^{\star} P<0.001$ versus low eGFP group in $\mathbf{e}, \mathbf{h}$ or versus low or medium mCherry group in $\mathbf{i}$. All electrophysiological data obtained at $37^{\circ} \mathrm{C}$, unless otherwise specified. Error bars indicate s.e.m; statistical significance was determined by one-way analysis of variance, followed by Tukey's post-hoc test to calculate $P$-values.

(Supplementary Fig. 7e-g), with cells adapting to higher pacing rates by decreasing $\mathrm{APD}_{80}$ and $\mathrm{CV}$ (Supplementary Fig. $7 \mathrm{~h}, \mathrm{i}$ ).

Applicability to other sources of human unexcitable cells. To further demonstrate the versatility of this approach, we used the Kir2.1- $\mathrm{Na}_{\mathrm{v}}$ SheP D60N lentivirus to stably convert human ventricular fibroblasts (HVFs), human astrocytes (HAs) and HEK293s into engineered electrically excitable cells (E-HVFs, E-HAs and E-HEK293, respectively). All engineered cell types displayed strong $I_{\mathrm{NaSheP}}$ and $I_{\mathrm{K} 1}$, and fired APs on stimulation (Supplementary Fig. 8). Expression of the Kir2.1 channels hyperpolarized the resting membrane potentials in all transduced cells to a similar value (Fig. $4 \mathrm{j}$ ), whereas similar $I_{\mathrm{NaSheP}}$ levels resulted in comparable upstroke velocities (Fig. 4k). Significant differences in AP duration among different cell types (Fig. 4l) could be attributed to differences in their endogenous currents (Fig. $4 \mathrm{~m}$ ) and potentially other differences in epigenetic, posttranscriptional and posttranslational regulation of ion channel expression and function. Collectively, these results confirmed the robustness of the $\mathrm{BacNa}_{\mathrm{v}}$ engineering approach against the starting unexcitable cell phenotype.

E-Fibs improve conduction in model of interstitial fibrosis. To test whether E-Fibs could improve impaired AP conduction in native excitable tissues, we designed a zig-zag micropattern of neonatal rat cardiomyocytes (Fig. 5a and Supplementary Fig. 9a), which mimics the tortuous electrical conduction of cardiac tissue with interstitial fibrosis ${ }^{24,25}$. The cardiomyocyte zig-zag micropattern displayed normal AP propagation in the longitudinal direction (Supplementary Fig. 9b), but significantly slowed down transverse CV $\left(3.2 \pm 0.4 \mathrm{~cm} \mathrm{~s}^{-1}\right.$; Supplementary Fig. 9c), which was fully rescued $\left(12.0 \pm 0.5 \mathrm{~cm} \mathrm{~s}^{-1}\right)$ by the electrical coupling of actively conducting E-HDFs and cardiomyocytes (Fig. 5b,c, Supplementary Fig. 9g and Supplementary video 3 ). In contrast, unexcitable wt-HDFs or excitable $\mathrm{Na}_{\mathrm{v}}$ SheP D60A + Kir2.1 HDFs coupled only by endogenous junctions (no Cx43 overexpression) did not alter the transverse conduction (Fig. $5 \mathrm{c}$ and Supplementary Fig. 9d,e), whereas unexcitable HDFs with overexpressed $\mathrm{Cx} 43$ yielded only a small conduction improvement (to $6.5 \pm 0.3 \mathrm{~cm} \mathrm{~s}^{-1}$; Fig. $5 \mathrm{c}$ and Supplementary Fig. 9f). All groups had similar AP durations (Fig. 5d); thus, wavelength differences (Fig. 5e) reflected the differences in CV. Importantly, these studies suggested that slow electrical propagation in cardiac tissues with interstitial fibrosis could be significantly improved if fibroblasts were engineered to actively conduct APs by becoming both excitable and robustly coupled.

BacNa $_{v}$ augment and rescue mammalian tissue excitability. Our computational results showed that select $\mathrm{BacNa}_{\mathrm{v}}$ mutant ( $\left.\mathrm{Na}_{\mathrm{v}} \mathrm{SheP} \mathrm{D} 60 \mathrm{~A}\right)$ displayed comparable AP upstroke and slightly lower $\mathrm{CV}$ than mammalian $\mathrm{Na}_{\mathrm{v}} 1.5$ channel in an excitable cell model (Fig. 2d,e), suggesting that in addition to custom design of de novo excitable cells and tissues, $\mathrm{BacNa}_{\mathrm{v}}$ channels could be exploited to directly manipulate or augment ${ }^{26,27}$ mammalian tissue excitability. Specifically, when lentivirally expressed in cardiomyocyte cultures, $\mathrm{Na}_{\mathrm{v}}$ SheP D60A channels significantly improved CV from $15.9 \pm 0.8$ to $26.1 \pm 0.7 \mathrm{~cm} \mathrm{~s}^{-1}$ without 
a

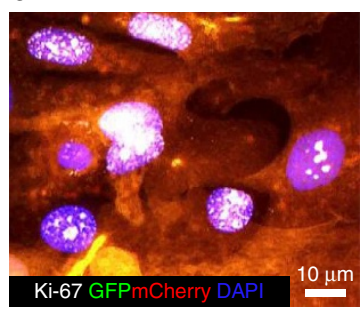

b

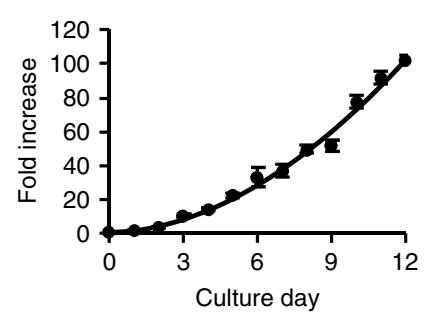

C

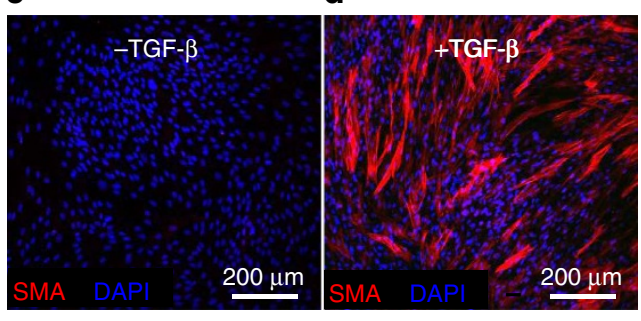

e

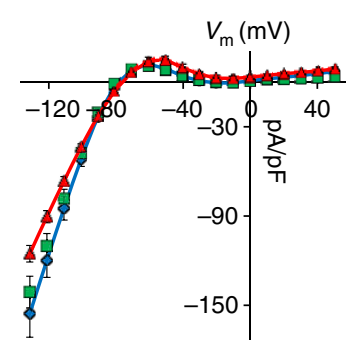

$\rightarrow$ Control f

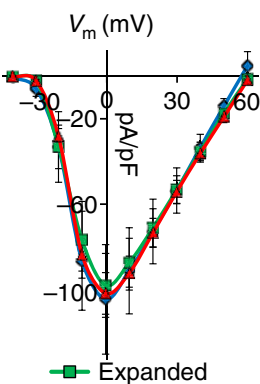

g

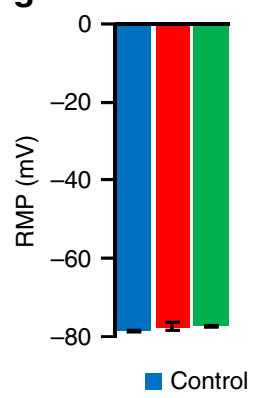

h

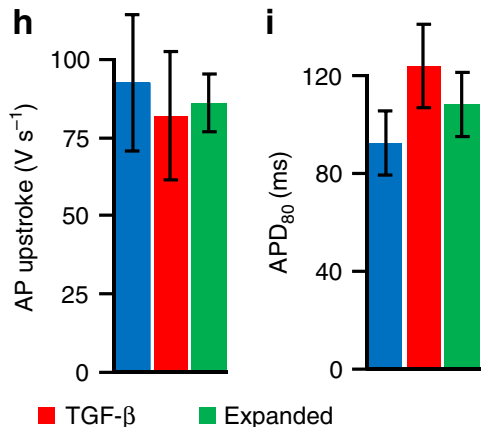

j

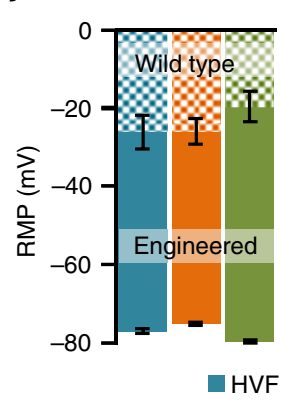

$\mathbf{k}$

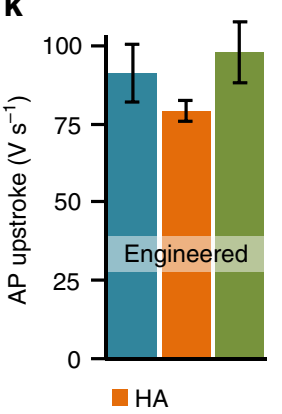

I 150

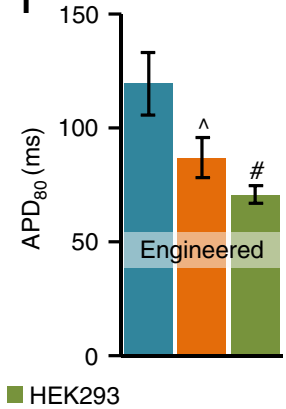

m

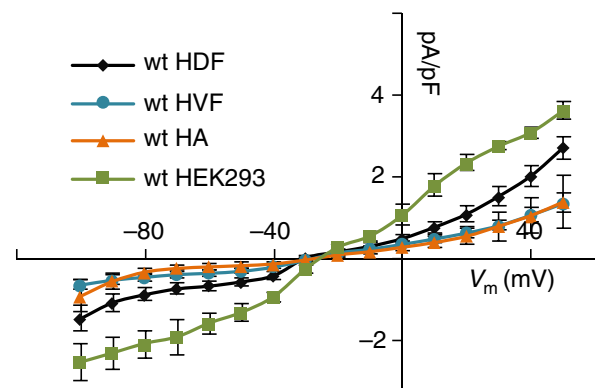

Figure 4 | Stability and robustness of E-Fib phenotype. (a,b) E-HDFs transduced with Na $\mathrm{v}_{\mathrm{v}}$ SheP D60N-P2A-eGFP-Kir2.1 and Cx43-P2A-mCherry lentiviruses retain proliferative potential as shown by Ki-67-positive staining (a) after $\sim 100$-fold expansion (b), 4 weeks post-lentiviral transduction. Growth curve is fit to a power function. (c,d) Conversion of smooth muscle actin (SMA)- E-HDFs (c) to SMA ${ }^{+}$myofibroblasts after transforming growth factor (TGF)- $\beta$ treatment (d). (e-i) Steady-state $I_{\mathrm{K} 1}-V(\mathbf{e})$ and peak $I_{\mathrm{Na}}-V$ (f) curves (recorded at $25^{\circ} \mathrm{C}$ ) as well as resting membrane potential $(R M P, \mathbf{g}), A_{80}(\mathbf{h})$ and AP upstroke velocity (i) do not differ among control, expanded or TGF- $\beta$-treated E-HDFs. $(n=5-9)$. (j-I) RMP (j), maximum $\mathrm{AP}$ upstroke velocity $(\mathbf{k})$ and $\mathrm{APD}_{80}(\mathbf{I})$ in different $\mathrm{E}-\mathrm{Fibs}(\mathrm{E}-\mathrm{HVF}$, human ventricular fibroblasts; $\mathrm{E}-\mathrm{HA}$, human astrocytes) transduced with the $\mathrm{Na}_{\mathrm{v}}$ SheP D60N-P2A-eGFP-Kir2.1 lentivirus $(n=6-8)$. ( $\left.\mathbf{m}\right)$ Steady-state $I-V$ curves in different wt Fibs $(n=4-6)$. ${ }^{\wedge} P<0.05, \# P<0.01$ versus HVF in $\mathbf{I}$. All electrophysiological data obtained at $37^{\circ} \mathrm{C}$, unless otherwise specified. Error bars indicate s.e.m; statistical significance was determined by one-way analysis of variance, followed by Tukey's post-hoc test to calculate $P$-values.

altering AP duration (Supplementary Fig. 10). Furthermore, expression of $\mathrm{BacNa}_{\mathrm{v}}$ channels could ameliorate reduced cardiac tissue excitability and arrhythmogenic conduction slowing in various pathological conditions (for example, ischaemia, infarction and congenital disease) associated with membrane depolarization and inactivation of $\mathrm{Na}_{\mathrm{v}} 1.5$ channels ${ }^{2,28,29}$ or diminished intercellular coupling ${ }^{30,31}$. For proof-of-concept studies, we used a $\mathrm{Na}_{\mathrm{v}}$ SheP D60A mutant, which exhibits $\sim 10 \mathrm{mV}$ more depolarized inactivation $V_{1 / 2}(-76.8 \mathrm{mV})$ compared with $\mathrm{Na}_{\mathrm{v}} 1.5$ channel $(-87.4 \mathrm{mV})$ (Fig. 6a,b). Elevating extracellular $\mathrm{K}^{+}$concentration to depolarize resting potential and inactivate Nav1.5 channels in Ex293 cells (a monoclonal HEK293 line engineered to stably express Kir2.1, $\mathrm{Cx} 43$ and $\mathrm{Na}_{\mathrm{v}} 1.5$ (ref. 32) resulted in conduction failure after addition of as little as $3 \mathrm{mM} \mathrm{KCl}$. In contrast, Ex293 monolayers transduced with the $\mathrm{Na}_{\mathrm{v}} \mathrm{SheP}$ D60A lentivirus sustained AP conduction at significantly higher extracellular $\mathrm{K}^{+}$ concentrations (up to $12.4 \mathrm{mM}$; Fig. 6c). Furthermore, $\mathrm{Na}_{\mathrm{v}} \mathrm{SheP}$ D60A expression in Ex293 monolayers subjected to increased doses of gap junctional blocker palmitoleic acid (PA) attenuated conduction slowing and prevented conduction block at $60 \mu \mathrm{M}$ PA when cells expressing only $\mathrm{Na}_{\mathrm{v}} 1.5$ channel failed to conduct (Fig. 6d). Finally, we tested whether viral expression of $\mathrm{Na}_{\mathrm{v}} \mathrm{SheP}$ D60A could increase the resistance to conduction failure in cardiomyocyte cultures that underwent regional ischaemia after being covered with a glass coverslip ${ }^{33}$ (Fig. 6e). During ischaemia, cardiomyocytes exhibited gradual conduction slowing (Fig. 6f,g) and APD shortening (Supplementary Fig. 11a,b), followed by the partial (Supplementary Fig. 11c) and eventually complete conduction block (Fig. 6h and Supplementary Video 4). Importantly, $\mathrm{Na}_{\mathrm{v}}$ SheP D60A expression preserved cell excitability to significantly prolong the time to conduction block (from $10.5 \pm 1.0 \mathrm{~min}$ in control to $14.2 \pm 0.7 \mathrm{~min}$ in $\mathrm{BacNa}_{\mathrm{v}^{-}}$ expressing cardiomyocytes; Fig. 6i). 

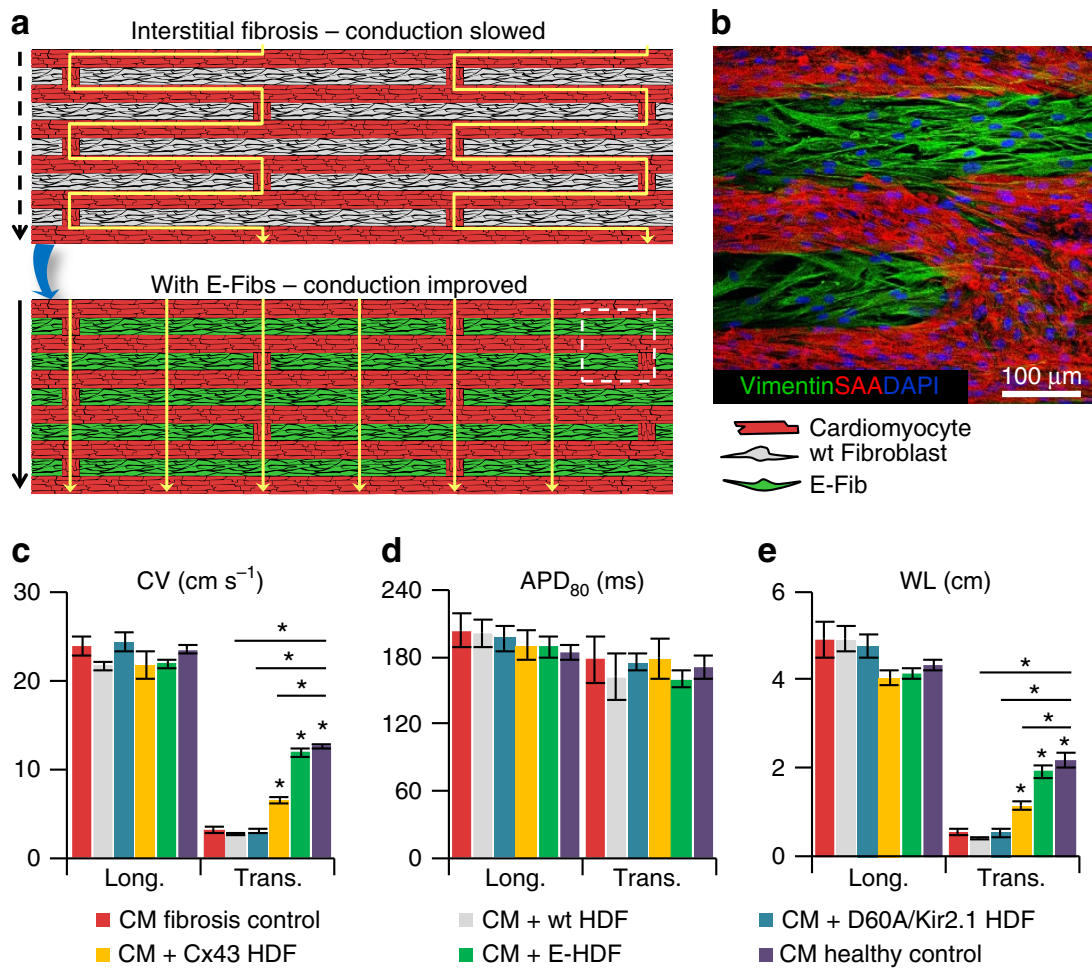

Figure 5 | Improvement of AP conduction by E-Fibs in a model of cardiac interstitial fibrosis. (a) Schematic depicting use of E-Fibs to improve impaired conduction in an in vitro model of cardiac interstitial fibrosis. (b) Immunostaining corresponding to dashed-line square area in a showing vimentin ${ }^{+}$E-HDFs in between sarcomeric $\alpha$-actinin (SAA) ${ }^{+}$neonatal rat cardiomyocytes (CMs). (c-e) Average CV (c), APD 80 (d) and impulse wavelength $\left(\mathrm{WL}=\mathrm{CVxAPD}{ }_{80}, \mathbf{e}\right)$ values during longitudinal or transverse conduction in $\mathrm{CM}$-only cultures (fibrosis control), $\mathrm{CM}+\mathrm{wt}-\mathrm{HDF}$ co-cultures, $\mathrm{CM}+\mathrm{Na} \mathrm{S}_{\mathrm{S}} \mathrm{S}$ P D60A/Kir2.1-expressing HDF co-cultures, CM + Cx43-expressing HDF co-cultures, CM + E-HDF co-cultures and confluent anisotropic CM cultures (healthy control) $(n=5-8) .{ }^{\star} P<0.001$ versus transverse $C V$ s in fibrosis control group. Error bars indicate s.e.m; statistical significance was determined by one-way analysis of variance, followed by Tukey's post-hoc test to calculate $P$-values.

\section{Discussion}

Since their discovery 15 years ago ${ }^{13}$, prokaryotic $\mathrm{Na}^{+}$channels have contributed greatly to understanding structure ${ }^{16,20}$, function $^{34,35}$ and pharmacology ${ }^{36}$ of the more complex eukaryotic VGSCs. Yet, it has remained unexplored if large diversity and small gene size of $\mathrm{BaCNa}_{\mathrm{v}}$ could be employed to stably modify and augment the excitability of mammalian tissues, a feat difficult to achieve using large mammalian VGSCs. In this study, we presented a combined experimental and computation strategy for use of $\mathrm{BacNa}_{\mathrm{v}}$ channels in the de novo induction and control of mammalian tissue excitability. Our goal was to develop a versatile platform that would be cost-effective, applicable to a variety of cell types and amenable to rapid production of large quantities of excitable cells for off-the-shelf use.

Previously, we applied a non-viral stepwise expression of cardiac $\mathrm{Na}^{+}$channel $\mathrm{Na}_{\mathrm{v}} 1.5$, inward rectifier $\mathrm{K}^{+}$channel Kir2.1 and gap junctional protein $\mathrm{Cx} 43$, combined with antibiotic selection, to generate a stable monoclonal HEK293 line capable of active AP conduction ${ }^{32}$. Other proof-of-concept studies applied adenoviral expressions of mammalian VGSCs to induce or modify excitability in primary tissues ${ }^{37-39}$; however, these effects were transient and unsuitable for long-term studies or the development of antiarrhythmic gene therapies ${ }^{38,39}$. Although the use of split-intein-mediated protein trans-splicing strategy ${ }^{40}$ might be adapted in the future to induce stable expression of mammalian VGSCs in primary cells, efficacy of this approach may be limited due to the need for co-transduction of two or more viruses to reconstitute functional channels. To circumvent the need for expressing large mammalian VGSCs, we exploited much smaller $\mathrm{BacNa}_{v}$ genes and designed high-titre multi-cistronic lentiviruses coding minimum sets of ion channels required for stable and efficient induction of excitability in primary cells. In fact, using $\mathrm{BacNa}_{\mathrm{v}}+\mathrm{Kir} 2.1$ lentiviruses, human and rodent fibroblasts were routinely transduced with 90\% efficiency without any loss of cell viability (not shown). Including a fluorescent reporter in the same virus allowed us to further purify transduced cells and fine-tune their electrical properties (Fig. 3 and Supplementary Fig. 5).

Importantly, lentivirally expressed ion currents in all engineered fibroblasts were much larger than their endogenous currents (Fig. 1). As such, resulting electrophysiological phenotype of engineered cells was relatively insensitive to the cell's epigenetic state and both predictable in silico and for the most part reproducible among different cell types in vitro (Fig. $4 \mathrm{j}-\mathrm{l}$ and Supplementary Fig. 8). The ability to generate actively conducting HVFs, in particular, suggested a potential therapeutic strategy whereby in situ fibroblast engineering could improve impaired conduction in fibrotic heart tissues. As a proof of concept, we showed that well-coupled and excitable (but not weakly coupled and/or unexcitable) fibroblasts successfully restored normal neonatal rat cardiomyocyte conduction in an in vitro model of interstitial fibrosis (Fig. 5). On the other hand, distinct forms of cardiac fibroses, differing in size, and cellular and non-cellular components can result from various types of heart disease or injury ${ }^{41}$. In situ engineering of actively conducting fibroblasts, while potentially beneficial in improving cardiac conduction in small regions of interstitial or diffuse fibrosis where these cells come in close contacts with cardiomyocytes, may not prove as effective in large cardiac scars with low cellularity. Of note is also that without the prominent $\mathrm{Ca}^{2}+$ currents, engineered fibroblasts 
a

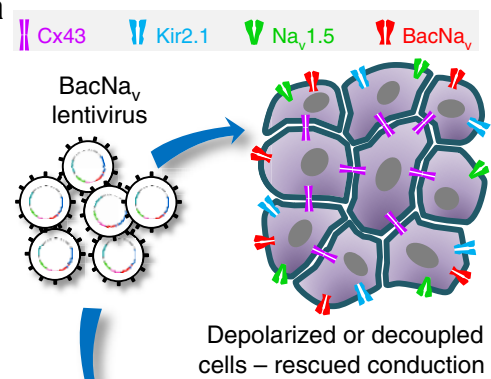

b

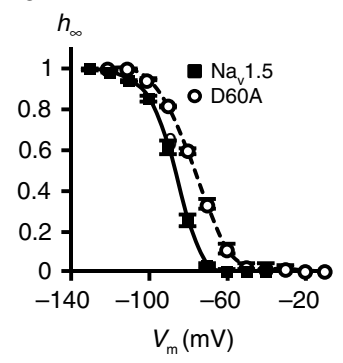

c

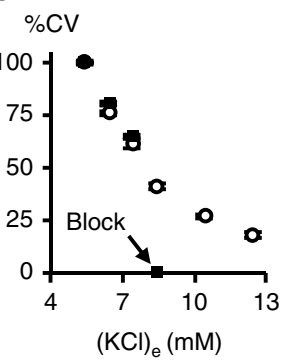

d

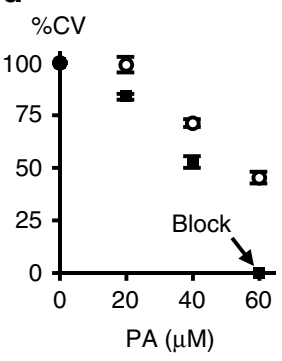

e

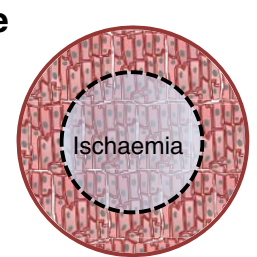

Ischaemic condition increased resistance to block

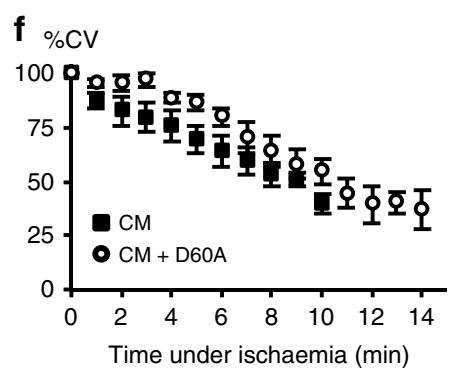

g

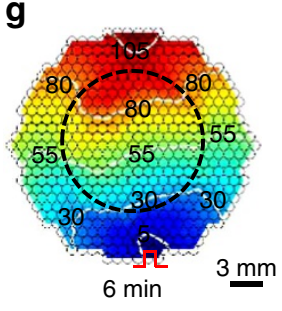

h

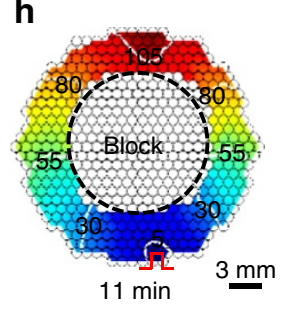

i

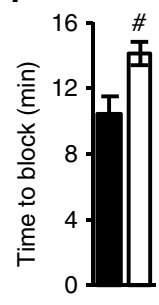

Figure 6 | Improvement of mammalian AP conduction by $\mathbf{B a c N a}$ in modeled pathological conditions. (a) Schematic depicting direct expression of $\mathrm{BacNa} \mathrm{a}_{\mathrm{v}}$ in mammalian excitable tissues containing endogenous $\mathrm{Na}_{\mathrm{v}} 1.5$, to enhance conduction and prevent conduction block in simulated pathological conditions. (b) Steady-state inactivation $\left(h_{\infty}\right)$ of $\mathrm{Na}_{\mathrm{v}}$ SheP D60A is more depolarized compared with that of $\mathrm{Na}_{\mathrm{v}} 1.5(n=5)$. (c,d) Conduction failure due to inactivation of $\mathrm{Na}_{v} 1.5$ by membrane depolarization with elevated extracellular $\mathrm{K}^{+}(\mathbf{c}, n=6)$ or blocking of intercellular coupling by PA (d, $\left.n=12-18\right)$ in Ex293 monolayers (black squares) is rescued by co-expression of $\mathrm{Na}_{v}$ SheP D60A (white circles). (e) Schematic depicting exogenous expression of BacNa in neonatal rat CMs to increase resistance to conduction block in ischaemic conditions. Black dashed circle denotes position of glass coverslip used to induce regional ischaemia in CM monolayer. (f) Progressive conduction slowing until block with time of ischaemia in control (CM) and $\mathrm{Na}$ SheP D60A transduced (CM + D60A) monolayers $(n=6)$. ( $(\mathbf{h}, \mathbf{h})$ Representative isochrones maps of a CM monolayer showing conduction slowing after 6 min $(\mathbf{g})$ and complete block after $11 \mathrm{~min}$ of ischaemia (h). Pulse signs indicate location of stimulating electrode. Circles denote 504 recording sites. (i) Under ischaemic condition, CMs transduced with $\mathrm{Na}_{v}$ SheP D60A lentivirus (white) resisted conduction block longer than control CMs (black) ( $n=6$, \#P<0.01). Error bars indicate s.e.m; statistical significance was determined by an unpaired Student's $t$-test to calculate $P$-value.

are unlikely to spontaneously fire APs or be a source of triggered activity, which could potentially make them less arrhythmogenic compared with the use of pluripotent stem cell-derived cardiomyocytes $^{42,43}$.

Furthermore, transduction of mammalian excitable tissue cultures with specific $\mathrm{BacNa}_{\mathrm{v}}$ mutants augmented or rescued conduction in healthy (Supplementary Fig. 10) and pathological (depolarization, decoupling and ischaemia; Fig. 6 and Supplementary Fig. 11) conditions, suggesting that $\mathrm{BacNa}_{\mathrm{v}}$-based gene therapies could be targeted directly to excitable cells. In addition, previous studies have shown that $\mathrm{BacNa}_{\mathrm{v}}$ could be converted into $\mathrm{Ca}^{2}{ }^{+}$-selective channel $\left(\mathrm{BacCa}_{\mathrm{v}}\right)$ by substituting three amino acid residues of the selectivity filter with Aspartate ${ }^{19,44,45}$. Hence, stable expression of engineered $\mathrm{BacNa}_{\mathrm{v}}$ channels or their $\mathrm{Ca}^{2+}$-selective derivatives may enable direct therapeutic effects on excitable and unexcitable cells whose function relies on voltage-gated $\mathrm{Na}^{+}$or $\mathrm{Ca}^{2+}$ channels.

Although in this study we focused on altering specific amino acid residues (E43 or D60) in a particular $\mathrm{BacNa}_{\mathrm{v}}$ orthologue $\left(\mathrm{Na}_{\mathrm{v}} \mathrm{SheP}\right)$, various combinations of other $\mathrm{BacNa}_{\mathrm{v}}$ orthologues and residues ${ }^{18,46,47}$ could be explored to customize phenotype of engineered or native excitable cells. For example, mutations in the 'glycine hinge' region in some $\mathrm{BacNa}_{\mathrm{v}}$ orthologues could change channel inactivation rate ${ }^{15}$, and along with tuning of Kir2.1 expression via an independent promoter, could be used to control AP duration to avoid arrhythmogenic dispersion of refractoriness if E-Fibs were used for cardiac cell or in situ gene therapy. Furthermore, replacing the highly conserved negative residues (E70 and D91) of $\mathrm{BacNa}_{\mathrm{v}}$ voltage-sensitive domain with positively charged residues could shift activation to more hyperpolarized voltages $^{48}$ and be used to reduce AP threshold. Similarly, substituting the negatively charged residues in the neck region of the carboxy-terminal domain (CTD) of the $\mathrm{BacNa}_{\mathrm{v}}$ channel into glycine could destabilize the helix conformation of this region, decrease the depolarization energy required for channel opening ${ }^{49}$ and further augment cell excitability and conduction. Furthermore, a recent study by Arrigoni et al. ${ }^{50}$ showed that $\mathrm{BacNa}_{\mathrm{v}}$ CTD plays a critical role in voltage dependence of activation, suggesting CTD swapping among different orthologues could provide yet another method to control excitability of engineered cells.

Basic studies in 'lower' organisms, including prokaryotes, have led to versatile approaches to manipulate and study mammalian biology. Recent examples include optogenetics ${ }^{51}$ and genomeediting techniques ${ }^{52}$ that, in addition to becoming powerful research tools, have been extensively investigated for potential medical applications. In line with these undertakings, our work supports expansion of prokaryotic ion channel research to basic studies of mammalian tissue excitability and development of new human therapies.

\section{Methods}

Screening of bacterial sodium channels. To engineer excitable cells, we first explored use of $\mathrm{NaChBac}$ channel, the longest studied $\mathrm{BacNa}_{\mathrm{v}}$ orthologue ${ }^{13,17,45,53}$ However, its slow recovery from inactivation yielded excitable cells with very low maximum capture rate $(<1 \mathrm{~Hz})$. This prompted us to instead use $\mathrm{Na}_{\mathrm{v}} \mathrm{RosD}_{\mathrm{G}} 217 \mathrm{~A}$ and $\mathrm{Na}_{\mathrm{v}} \mathrm{SheP}$ channels that were kindly provided by Dr Katsumasa Irie from Kyoto University $^{15}$. Libraries of SheP(D60X) and SheP(E43X) mutants were generated using Quikchange II XL Site-Directed Mutagenesis Kit (Agilent Technologies), as 
per the manufacturer's instructions. All the primers used are listed in Supplementary Table 2. Each gene was subcloned into the mammalian expression vector pCMV5(CuO)-IRES-GFP (Qbiogene) and transfected into HEK293 cells stably expressing Kir2.1 using Lipofectamine 2000 transfection reagent (Life Technologies). GFP fluorescence was usually detected after $12 \mathrm{~h}$ and cells were patch clamped $24 \mathrm{~h}$ after transfection, to derive AP parameters.

Lentivirus production. Lentiviral plasmids were constructed from the pRRL-CMV vector (a gift from Dr Inder Verma, Salk Institute). $\mathrm{BaCNa}_{\mathrm{v}}\left(\mathrm{Na}_{\mathrm{v}} \mathrm{RosD}\right.$ G217A or $\mathrm{Na}_{\mathrm{v}}$ SheP mutant, without a stop codon) was ligated with eGFP-Kir2.1 (Kir2.1 fused with eGFP at the amino terminus) via the viral P2A peptide ${ }^{54}$ before being subcloned into pRRL-CMV, to create the bi-cistronic lentiviral plasmid pRRL-CMV-BacNa $a_{\mathrm{v}}$-P2A-eGFP-Kir2.1. To enhance gap junction expression in engineered cells, $\mathrm{Cx} 43$ (ref. 32) was linked with mCherry via P2A and subcloned into the pRRL-CMV lentiviral plasmid (pRRL-CMV-Cx43-P2A-mCherry). In the main text, $\mathrm{BacNa}_{\mathrm{v}}$-Kir2.1 and $\mathrm{Cx} 43$ lentiviruses refer to pRRL-CMV-BacNa $\mathrm{v}_{\mathrm{v}}{ }^{-}$ P2A-eGFP-Kir2.1 and pRRL-CMV-Cx43-P2A-mCherry virus, respectively. Other lentiviral constructs used in the study were pRRL-CMV-BacNa $a_{\mathrm{v}}-\mathrm{P} 2 \mathrm{~A}-\mathrm{Kir} 2.1$ T2A-eGFP and pRRL-CMV-BacNav - T2A-eGFP. High-titre lentiviruses were produced using second generation lentiviral packaging system. Briefly, 293FT cells (Life Technologies, R700-07) were co-transfected with lentiviral plasmid, packaging plasmid psPAX2 and envelope plasmid pMD2.G (2:1:1 mass ratios) using Lipofectamine 2000 (Life Technologies). Supernatant containing lentiviral particles was collected $72 \mathrm{~h}$ after transfection, centrifuged $(500 \mathrm{~g}, 10 \mathrm{~min})$ and filtered through $0.45 \mu \mathrm{m}$ cellulose acetate filter (Corning) to remove cell debris before combined with Lenti-X Concentrator (Clontech) at 3:1 volume ratio for overnight incubation at $4{ }^{\circ} \mathrm{C}$. Concentrated lentiviral particles were harvested following 45 min centrifugation $\left(1,500 \mathrm{~g}, 4^{\circ} \mathrm{C}\right)$ and resuspended in $1 / 10$ to $1 / 100$ of the original volume in DMEM medium. Plasmids psPAX2 and pMD2.G were obtained from Didier Trono (Addgene plasmids \#12260 and \#12259).

\section{Generation of engineered human fibroblasts. Primary adult HDFs} (Gibco, C-013-5C) were co-transduced with $\mathrm{BacNa}_{\mathrm{v}}$-Kir2.1 and Cx43 lentiviruses at a density of $10^{4}$ cells per $\mathrm{cm}^{2}$ in fibroblast basal medium (ATCC, PCS-201-030). Five days after lentiviral transduction, $\mathrm{GFP}^{+} / \mathrm{mCherry}^{+}$cells were sorted by FACS analysis, replated in fibroblast growth medium (basal medium supplemented with fibroblast low-serum growth kit, ATCC, PCS-201-041) and assessed with patch-clamp recordings and optical mapping at 1 week and 4 weeks after sorting. Conversion of HDFs into myofibroblasts was induced by adding transforming growth factor- $\beta\left(10 \mathrm{ng} \mathrm{m}^{1-1}\right)$ into culture media for 5 days. Similar lentiviral transduction protocol was repeated in HVFs (Lonza, CC-2904), HAs (Lonza, CC-2565) and HEK293 cells (ATCC, CRL-1573).

Isotropic and anisotropic monolayers of HDFs. E-HDFs were seeded onto $22 \mathrm{~mm}$-diameter Aclar (Electron Microscopy Sciences) coverslips coated with fibronectin (Sigma, $15 \mu \mathrm{g} \mathrm{ml}^{-1}, 30 \mathrm{~min}$ ) at a density of $5 \times 10^{4}$ cells per $\mathrm{cm}^{2}$ to form isotropic monolayers. Anisotropic E-HDF monolayers were formed on Aclar coverslips microcontact printed with a pattern of parallel fibronectin lines $\left(10 \mu \mathrm{m}\right.$ width, $10 \mu \mathrm{m}$ spacing) as previously described ${ }^{55}$. Cells were cultured for $24 \mathrm{~h}$ to become confluent and form intercellular junctions followed by optical mapping of AP propagation and immunostaining.

Micropatterned zig-zag cultures. All animals were treated in accordance with protocols approved by the Duke University Institutional Animal Care and Use Committee. Neonatal rat cardiomyocytes were enzymatically dissociated from ventricles of 2-day-old Sprague-Dawley rats and enriched using differential pre-plating ${ }^{56}$. Specifically, ventricles were excised, minced and incubated with $0.1 \%$ trypsin overnight and dissociated in four sequential steps using $0.1 \%$ collagenase. Isolated cells were centrifuged $(5 \mathrm{~min}, 200 \mathrm{~g}$ ) and further enriched by two $45 \mathrm{~min}$ pre-plating steps to select for cardiomyocyte-enriched cells that remained unattached after the second pre-plating. Zig-zag cultures were generated as previously described ${ }^{25}$. Briefly, Aclar coverslips (22 mm diameter) were coated with $5 \mu \mathrm{g} \mathrm{ml}^{-1}$ collagen I (rat tail, BD Biosciences) for $30 \mathrm{~min}$, rinsed with PBS, then micro-contact printed with a zig-zag network of fibronectin $(100 \mu \mathrm{m}$-wide lines spaced $100 \mu \mathrm{m}$ apart and connected by $100 \mu \mathrm{m}$-wide transverse bridges staggered every $6 \mathrm{~mm}$ ). Cardiomyocytes were seeded at a density of $2 \times 10^{5}$ cells per $\mathrm{cm}^{2}$ in DMEM/F-12 medium (Gibco, 11320-033) supplemented with $10 \%$ calf serum and $10 \%$ horse serum, followed by a switch to $5 \%$ fetal bovine serum media at culture day 2. The following day, E-HDFs were added to the cardiomyocyte zig-zag cultures at a density of $2.5 \times 10^{4}$ cells per $\mathrm{cm}^{2}$. On day 5 , co-cultured monolayers were assessed for AP propagation by optical mapping and subsequently fixed for immunostaining.

Immunostaining. Cell monolayers or tissue patches were fixed in 2\% paraformaldehyde $(15 \mathrm{~min})$, permeabilized in $0.5 \%$ Triton-X $(30 \mathrm{~min})$ and blocked in a $5: 1$ solution of $1 \% \mathrm{BSA}$ and chicken serum $(30 \mathrm{~min})$. The following primary antibodies ( $1 \mathrm{~h}$ incubation) were used: anti-sarcomeric $\alpha$-actinin (Sigma, a7811, 1:200), anti-Cx43 (Life Technologies, 71-0700, 1:100), anti-vimentin (Sigma, v6630,
1:500), anti-smooth muscle actin (Sigma, a2547, 1:200), anti-GFAP (BD Biosciences, 561483, 1:100) and anti-Ki67 (Abcam, ab15580, 1:200). Secondary antibodies ( $1 \mathrm{~h}$ incubation) included the following: Alexa488 (Life Technologies, A-21200 or A-21441, 1:200), Alexa594 (Life Technologies, A-21201 or A-21442, 1:200), Alexa647 (Life Technologies, A-21463, 1:200), Alexa488-conjugated phalloidin (Life Technologies, A12379, 1:300) and 4,6-diamidino-2-phenylindole (Sigma, 1:300). All immunostaining steps were performed at room temperature. Fluorescence images were acquired using inverted fluorescence (Nikon TE2000) or confocal (Leica SP5) microscope and processed with ImageJ software.

Fluorescence recovery after photobleaching. Functional coupling in wt and Cx43-expressing HDFs was assessed using fluorescence recovery after photobleaching ${ }^{21,57}$ as previously described ${ }^{58}$. Briefly, confluent HDF monolayers were stained with Calcein AM dye (Molecular Probes, $0.5 \mu \mathrm{M}$ in DMEM) for $20 \mathrm{~min}$ at $37^{\circ} \mathrm{C}$, washed with PBS and immersed again in fresh media. The target cell was photobleached with a $488 \mathrm{~nm}$ Argon laser and subsequently imaged every $15 \mathrm{~s}$ for 6 min using an upright confocal microscope (Zeiss, LSM 510). The level of fluorescence in the target cell was normalized to the fluorescence level in remote unbleached cells and plot as a function of time post photobleaching.

Whole-cell patch-clamp recordings. Cells were briefly trypsinized, plated onto poly-L-lysine-coated glass coverslips and left to attach for $5 \mathrm{~h}$. Cells were then transferred to a patch-clamp chamber perfused with Tyrode's solution containing (in mM): $135 \mathrm{NaCl}$, $5.4 \mathrm{KCl}, 1.8 \mathrm{CaCl}_{2}, 1 \mathrm{MgCl}_{2}, 0.33 \mathrm{NaH}_{2} \mathrm{PO}_{4}, 5$ HEPES and 5 glucose. Patch pipettes were fabricated with tip resistances of $1-2 \mathrm{M} \Omega$ when filled with pipette solution consisting of (in $\mathrm{mM}$ ): $140 \mathrm{KCl}, 10 \mathrm{NaCl}, 1 \mathrm{CaCl}_{2}, 2 \mathrm{MgCl}_{2}$ 10 EGTA, 10 HEPES and 5 MgATP. Whole-cell voltage-clamp and current-clamp recordings were acquired at room temperature $\left(25^{\circ} \mathrm{C}\right)$ and $37^{\circ} \mathrm{C}$, respectively, using the Multiclamp 700B amplifier (Axon Instruments), filtered with a $10-\mathrm{kHz}$ Bessel filter, digitized at $40 \mathrm{kHz}$ and analysed using WinWCP software (John Dempster, University of Strathclyde). To measure $\mathrm{BaCNa}_{\mathrm{v}}$ activation properties, membrane voltage was stepped from a holding potential of $-100 \mathrm{mV}$ to varying $500 \mathrm{~ms}$ test potentials $(-50$ to $60 \mathrm{mV}$, increments of $10 \mathrm{mV}$ ). Inactivation of $\mathrm{BaCNa}_{\mathrm{v}}$ was derived from peak currents measured at $0 \mathrm{mV}$ after varying $3 \mathrm{~s}$ prepulse potentials ( -160 to $-30 \mathrm{mV}$, increments of $10 \mathrm{mV}$ ). For the channels with more hyperpolarized activation and inactivation $\left(\mathrm{Na}_{\mathrm{v}} \mathrm{SheP} \mathrm{WT}\right.$ and D60E), holding voltage for steady-state activation protocol was set at $-160 \mathrm{mV}$, whereas steady-state inactivation protocol included prepulse potentials from $-180 \mathrm{mV}$ to $-30 \mathrm{mV}$. Steady-state $I_{\mathrm{K} 1}-V$ curve was constructed from the current responses to varying $1 \mathrm{~s}$ test potentials $(-130$ to $50 \mathrm{mV}$, increments of $10 \mathrm{mV}$ ) from a holding potential of $-40 \mathrm{mV}$. APs were elicited by injecting a $1 \mathrm{~ms}$ current pulse at $1.1 \times$ threshold amplitude.

Optical mapping of AP propagation. Cell monolayers or zig-zag cultures were optically mapped with a $20 \mathrm{~mm}$ diameter hexagonal array of 504 optical fibres (Redshirt Imaging), as previously described ${ }^{32,55,56}$. Specifically, cultures were stained with a voltage-sensitive dye (Di-4-ANEPPS, $10 \mu \mathrm{M}$ ) for $5 \mathrm{~min}$ at room temperature, transferred to a recording chamber filled with Tyrode's solution at $37^{\circ} \mathrm{C}$ and illuminated by a solid-state excitation light source (Lumencor, SOLA SM) filtered through a $520 \pm 30 \mathrm{~nm}$ bandpass filter. Emitted red fluorescence signals $(\lambda>590 \mathrm{~nm})$ were collected by the optical fiber array, converted to voltage signals by photodiodes and recorded at a $2.4 \mathrm{kHz}$ sampling rate with a $750 \mu \mathrm{m}$ spatial resolution. AP propagation was triggered by a $10 \mathrm{~ms}, 1.2 \times$ threshold, $1 \mathrm{~Hz}$ stimulus from either a bipolar point electrode (in isotropic or anisotropic monolayers of E-HDFs) or a line electrode (in the zig-zag cultures). Generation of isochrone maps and calculation of $\mathrm{CV}, \mathrm{AP}$ duration at $80 \%$ repolarization $\left(\mathrm{APD}_{80}\right)$ and impulse wavelength were performed using custom MATLAB software, as previously described ${ }^{59,60}$

Depolarization and decoupling studies. Similar to previous studies ${ }^{61}$, membrane depolarization in Ex293 monolayers was induced by applying increasing doses of $\mathrm{KCl}$ (in $1 \mathrm{mM}$ increments starting from $5.4 \mathrm{mM}$ ). After 5 min equilibration at each $\mathrm{KCl}$ dose, cells were paced at $1 \mathrm{~Hz}$ and AP conduction optically mapped. For gap junction decoupling studies, PA (Sigma, 76168) was dissolved in dimethyl sulfoxide and sonicated $\left(10 \mathrm{~min}, 37^{\circ} \mathrm{C}\right)$ to yield a stock concentration of $50 \mathrm{mM}$. Ex293 monolayers were exposed to increasing doses of PA $(0,20,40$ and $60 \mu \mathrm{M})$ and after 15 min equilibration at each dose, cells were paced at $1 \mathrm{~Hz}$ and AP conduction optically mapped.

Regional ischaemia studies. Regional ischaemia in neonatal rat cardiomyocyte monolayers was induced as described previously ${ }^{33,62}$. Briefly, $12 \mathrm{~mm}$ round glass coverslip was placed onto the central area of a $22 \mathrm{~mm}$ cardiac monolayer to induce regional ischaemia. Cells were continuously paced at $1 \mathrm{~Hz}$ and AP conduction optically mapped every $1 \mathrm{~min}$ during ischaemia, until complete conduction failure in the ischaemic region. $\mathrm{CV}, \mathrm{APD}_{80}$ and optical $\mathrm{AP}$ amplitude measured at the recording sites of the central ischemic region were normalized to corresponding baseline values before ischemia. Recording site was defined as inactive (not conducting) if its AP amplitude failed below $10 \%$ of baseline value. 
Computational modelling. Experimentally derived modelling parameters are listed in Supplementary Table 3. The AP generation in HEK293 cells co-expressing $\mathrm{K}_{\mathrm{ir}} 2.1$ and $\mathrm{BacNa}_{\mathrm{v}}$ channels was simulated using the Hodgkin-Huxley formalism ${ }^{63}$ and the following differential equation:

$$
\frac{\mathrm{d} V}{\mathrm{~d} t}=-\frac{1}{C_{\mathrm{m}}}\left(I_{\mathrm{Na}}(V, t)+I_{\mathrm{K} 1}(V)+I_{\text {endo }}(V)-I_{\mathrm{stim}}(V, t)\right)
$$

where $V$ is the cell transmembrane potential, $C_{\mathrm{m}}$ is the total membrane capacitance, $I_{\mathrm{Na}}$ is the $\mathrm{BacNa}_{\mathrm{v}}$ channel current, $I_{\mathrm{K} 1}$ is the inward-rectifier potassium current, $I_{\text {endo }}$ is the endogenous background current and $I_{\text {stim }}$ is the stimulus current. Based on the similarities between $\mathrm{BacNa}_{\mathrm{v}}$ and mammalian $\mathrm{Ca}_{\mathrm{v}}$ channels ${ }^{13,45}$, the $\mathrm{BaCNa}_{\mathrm{v}}$ conductance was modelled as a simple product of activation and inactivation gating variables and $I_{\mathrm{Na}}$ was expressed using the following equation:

$$
I_{\mathrm{Na}}(V, t)=\overline{\mathrm{G}}_{\mathrm{Na}} * m(V, t) * h(V, t) *\left(V-E_{\mathrm{Na}}\right)
$$

where $\overline{\mathrm{G}}_{\mathrm{Na}}$ is the maximum conductance of the $\mathrm{BacNa}_{\mathrm{v}}$ channel, $m$ and $h$ are the first-order activation and inactivation gating variables, respectively, and $E_{\mathrm{Na}}$ is the sodium Nernst potential given in Supplementary Table 3. Mathematical model of human $\mathrm{Na}_{\mathrm{v}} 1.5$ was adopted from a previous model of human ventricular tissue ${ }^{64}$ with a slightly modified inactivation time constant to better fit voltage clamp recordings in $\mathrm{Na}_{\mathrm{v}} 1.5$-expressing HEK293 cells.

From the voltage-clamp activation and inactivation protocols, the steady-state values of the gating variables as a function of $\mathrm{V}$ were plotted and fit to the Boltzmann equation ${ }^{63}$, to derive the midpoint voltage and slope factor listed in Supplementary Table 1:

$$
m_{\infty}=\frac{1}{1+\exp \left(\frac{V_{1 / 2}-V}{\text { Slope }}\right)} h_{\infty}=\frac{1}{1+\exp \left(\frac{V-V_{1 / 2}}{\text { Slope }}\right)}
$$

The initial and late phases of $I_{\mathrm{Na}}$ traces for each clamp potential were fit as single exponentials to derive time constants of activation $\left(\tau_{\mathrm{m}}\right)$ and inactivation $\left(\tau_{\mathrm{h}}\right)$ as functions of $V$. The $\tau_{\mathrm{m}}$ and $\tau_{\mathrm{h}}$ at $V=+20 \mathrm{mV}$ are listed in Supplementary Table 1.

Voltage-clamp recordings of inward-rectifying potassium current were used to fit steady-state $I_{\mathrm{K} 1}$ values to a time-independent Nygren et al. ${ }^{65}$ model as follows:

$$
I_{\mathrm{K} 1}=\overline{\mathrm{G}}_{\mathrm{K} 1} \frac{V-E_{\mathrm{K}}}{1+e^{0.08754\left(V-E_{\mathrm{K}}-18.87\right)}}
$$

where $\overline{\mathrm{G}}_{\mathrm{K} 1}$ is the maximum conductance of the $\mathrm{K}_{\mathrm{ir}} 2.1$ channel and $E_{\mathrm{K}}$ is the potassium Nernst potential given in Supplementary Table 3.

Finally, the endogenous background current of wt HEK293 or HDF cells, $I_{\text {endo }}$ (in $\mathrm{pA}$ ), was fit by a time-independent linear equation:

$$
I_{\text {endo }}=2.6 \mathrm{~V}+12.3\left(\text { forHEK293) or } I_{\text {endo }}=0.53 \mathrm{~V}+17.5\right. \text { (forHDF) }
$$

where $V$ is given in millivolts. Simulations of single-cell APs for different $\mathrm{BacNa}_{\mathrm{v}}$ mutants were performed using constant $\overline{\mathrm{G}}_{\mathrm{K} 1}$ and $\overline{\mathrm{G}}_{\mathrm{Na}}$ values (measured by patch clamp in a monoclonal HEK293 line expressing $\mathrm{K}_{\mathrm{ir}} 2.1, \mathrm{Cx} 43$ and $\mathrm{Na}_{\mathrm{v}} \mathrm{SheP}$ D60A; Supplementary Table 3), to allow direct comparisons of how kinetic properties of $\mathrm{BacNa}_{\mathrm{v}}$ on their own affect AP characteristics (upstroke velocity, $\mathrm{APD}_{80}$ and threshold potential). As the whole-cell voltage clamp recordings were performed at $25^{\circ} \mathrm{C}$, to model AP generation at $37^{\circ} \mathrm{C}$, we scaled up $E_{\mathrm{K}}$ and $E_{\mathrm{Na}}$ from Supplementary Table 3 by $310.15^{\circ} \mathrm{K} / 298.15^{\circ} \mathrm{K}$ and further scaled $I_{\mathrm{Na}}, I_{\mathrm{K} 1}$ and $I_{\text {endo }}$ using $Q$-values of $2.7,1.8$ and 2.1, respectively.

To simulate AP propagation in HEK293 cells expressing different $\mathrm{BaCNa}_{\mathrm{v}}$ mutants, we applied a continuous cable equation discretized using forward Euler's method $^{66-68}$ as follows:

$v_{\mathrm{m}}(i+1, j)=v_{\mathrm{m}}(i, j)+\frac{a \Delta t}{2 R_{\mathrm{i}} c_{\mathrm{m}}}\left(\frac{v_{\mathrm{m}}(i, j+1)-2 v_{\mathrm{m}}(i, j)+v_{\mathrm{m}}(i, j-1)}{\Delta x^{2}}\right)-\frac{\Delta t}{c_{\mathrm{m}}} \sum J_{\text {ion }}(i, j)$

where $\Delta t$ and $\Delta x$ are the time (index $i$ ) and space (index $j$ ) discretization steps, respectively, $c_{\mathrm{m}}$ is the specific membrane capacitance, $a$ is the cable radius, $R_{\mathrm{i}}$ is the intracellular resistivity, and $\mathrm{J}_{\text {ion }}=\mathrm{J}_{\mathrm{Na}}+\mathrm{J}_{\mathrm{K} 1}+\mathrm{J}_{\text {endo }}$ is the total ionic current density (in $\left.\mathrm{pA} \mathrm{cm}{ }^{-2}\right)$, calculated by dividing the total ionic current $\left(\mathrm{I}_{\mathrm{Na}}+\mathrm{I}_{\mathrm{K} 1}+\mathrm{I}_{\mathrm{endo}}\right)$ from equation (1) with total membrane surface area. The $R_{\mathrm{i}}$ value was adopted to simulate the mean CV measured in optically mapped monolayers of a monoclonal HEK293 line expressing $\mathrm{K}_{\mathrm{ir}} 2.1$, $\mathrm{Cx} 43$ and $\mathrm{Na}_{\mathrm{v}}$ SheP D60A. All simulations were performed with the identical model parameters given in Supplementary Table 3 and distinct kinetic properties measured in different $\mathrm{BaCNa}_{\mathrm{v}}$ mutants (adjusted to $37^{\circ} \mathrm{C}$ as described above).

The total length of the simulated cable was $0.72 \mathrm{~cm}$ (that is, 300 nodes). The first three nodes in the cable were stimulated by current injection $(1.2 \times$ threshold $)$ at $1 \mathrm{~Hz}$ rate and activation time at each node was determined as the time of maximum upstroke velocity of propagated AP. Inverse slope of activation time versus distance line fit in the central $0.24 \mathrm{~cm}$ of the cable was used to calculate CV.

Statistical analysis. All data are presented as mean \pm s.e.m. and statistical significance was determined by one-way analysis of variance, followed by Tukey's post-hoc test to calculate $P$-values. Statistical significance was defined as ${ }^{\wedge} P<0.05$, ${ }^{\#} P<0.01$ and ${ }^{\star} P<0.0001$.
Data availability. All data supporting the findings of this study are available within the article and its Supplementary Information files or can be requested from the authors.

\section{References}

1. Catterall, W. A., Goldin, A. L. \& Waxman, S. G. International Union of Pharmacology. XLVII. Nomenclature and structure-function relationships of voltage-gated sodium channels. Pharmacol. Rev. 57, 397-409 (2005).

2. George, A. L. Jr Inherited disorders of voltage-gated sodium channels. J. Clin Invest. 115, 1990-1999 (2005).

3. Weiss, J. et al. Loss-of-function mutations in sodium channel Nav1.7 cause anosmia. Nature 472, 186-190 (2011).

4. Lossin, C. et al. Epilepsy-associated dysfunction in the voltage-gated neuronal sodium channel SCN1A. J. Neurosci. 23, 11289-11295 (2003).

5. Kamiya, K. et al. A nonsense mutation of the sodium channel gene SCN2A in a patient with intractable epilepsy and mental decline. J. Neurosci. 24, 2690-2698 (2004).

6. Sanders, S. J. et al. De novo mutations revealed by whole-exome sequencing are strongly associated with autism. Nature 485, 237-241 (2012).

7. Kapplinger, J. D. et al. An international compendium of mutations in the SCN5A-encoded cardiac sodium channel in patients referred for Brugada syndrome genetic testing. Heart Rhythm 7, 33-46 (2010).

8. $\mathrm{Hu}, \mathrm{D}$. et al. Mutations in SCN10A are responsible for a large fraction of cases of Brugada syndrome. J. Am. Coll. Cardiol. 64, 66-79 (2014).

9. Jurkat-Rott, K. et al. Voltage-sensor sodium channel mutations cause hypokalemic periodic paralysis type 2 by enhanced inactivation and reduced current. Proc. Natl Acad. Sci. USA 97, 9549-9554 (2000).

10. Bulman, D. E. et al. A novel sodium channel mutation in a family with hypokalemic periodic paralysis. Neurology 53, 1932-1936 (1999).

11. Black, J. A. \& Waxman, S. G. Noncanonical roles of voltage-gated sodium channels. Neuron 80, 280-291 (2013).

12. Catterall, W. A. From ionic currents to molecular mechanisms: the structure and function of voltage-gated sodium channels. Neuron 26, 13-25 (2000).

13. Ren, D. et al. A prokaryotic voltage-gated sodium channel. Science 294, 2372-2375 (2001).

14. Koishi, R. et al. A superfamily of voltage-gated sodium channels in bacteria. J. Biol. Chem. 279, 9532-9538 (2004).

15. Irie, K. et al. Comparative study of the gating motif and C-type inactivation in prokaryotic voltage-gated sodium channels. J. Biol. Chem. 285, 3685-3694 (2010).

16. Payandeh, J., Scheuer, T., Zheng, N. \& Catterall, W. A. The crystal structure of a voltage-gated sodium channel. Nature 475, 353-358 (2011)

17. Charalambous, K. \& Wallace, B. A. NaChBac: the long lost sodium channel ancestor. Biochemistry 50, 6742-6752 (2011).

18. Payandeh, J. \& Minor, D. L. Jr Bacterial Voltage-Gated Sodium Channels (BacNas) from the Soil, Sea, and Salt Lakes Enlighten Molecular Mechanisms of Electrical Signaling and Pharmacology in the Brain and Heart. J. Mol. Biol. 427, 3-30 (2014).

19. Shaya, D. et al. Voltage-gated sodium channel $(\mathrm{NaV})$ protein dissection creates a set of functional pore-only proteins. Proc. Natl Acad. Sci. USA 108, 12313-12318 (2011).

20. Zhang, X. et al. Crystal structure of an orthologue of the NaChBac voltagegated sodium channel. Nature 486, 130-134 (2012).

21. Wade, M. H., Trosko, J. E. \& Schindler, M. A fluorescence photobleaching assay of gap junction-mediated communication between human cells. Science 232, 525-528 (1986).

22. Shimomura, T., Irie, K., Nagura, H., Imai, T. \& Fujiyoshi, Y. Arrangement and mobility of the voltage sensor domain in prokaryotic voltage-gated sodium channels. J. Biol. Chem. 286, 7409-7417 (2011).

23. Martin, P. Wound healing--aiming for perfect skin regeneration. Science 276, 75-81 (1997).

24. de Bakker, J. et al. Slow conduction in the infarcted human heart. 'Zigzag' course of activation. Circulation 88, 915-926 (1993).

25. Kirkton, R. D. \& Bursac, N. Genetic engineering of somatic cells to study and improve cardiac function. Europace 14(Suppl 5): v40-v49 (2012).

26. Lin, C. W. et al. Genetically increased cell-intrinsic excitability enhances neuronal integration into adult brain circuits. Neuron 65, 32-39 (2010).

27. Bando, Y. et al. Control of spontaneous $\mathrm{Ca}^{2+}$ transients is critical for neuronal maturation in the developing neocortex. Cereb. Cortex 26, 106-117 (2014).

28. Janse, M. J. \& Wit, A. L. Electrophysiological mechanisms of ventricular arrhythmias resulting from myocardial ischemia and infarction. Physiol. Rev. 69, 1049-1169 (1989).

29. Carmeliet, E. Cardiac ionic currents and acute ischemia: from channels to arrhythmias. Physiol. Rev. 79, 917-1017 (1999).

30. Severs, N. J., Bruce, A. F., Dupont, E. \& Rothery, S. Remodelling of gap junctions and connexin expression in diseased myocardium. Cardiovasc. Res. 80, 9-19 (2008). 
31. Danik, S. B. et al. Modulation of cardiac gap junction expression and arrhythmic susceptibility. Circ. Res. 95, 1035-1041 (2004).

32. Kirkton, R. D. \& Bursac, N. Engineering biosynthetic excitable tissues from unexcitable cells for electrophysiological and cell therapy studies. Nat. Commun. 2, 300 (2011).

33. de Diego, C. et al. Electrophysiological consequences of acute regional ischemia/reperfusion in neonatal rat ventricular myocyte monolayers. Circulation 118, 2330-2337 (2008).

34. McCusker, E. C. et al. Structure of a bacterial voltage-gated sodium channel pore reveals mechanisms of opening and closing. Nat. Commun. 3, 1102 (2012).

35. Bagneris, C., Naylor, C. E., McCusker, E. C. \& Wallace, B. A. Structural model of the open-closed-inactivated cycle of prokaryotic voltage-gated sodium channels. J. Gen. Physiol. 145, 5-16 (2015).

36. Bagneris, C. et al. Prokaryotic NavMs channel as a structural and functional model for eukaryotic sodium channel antagonism. Proc. Natl Acad. Sci. USA 111, 8428-8433 (2014).

37. Hou, L., Hu, B. \& Jalife, J. Genetically engineered excitable cardiac myofibroblasts coupled to cardiomyocytes rescue normal propagation and reduce arrhythmia complexity in heterocellular monolayers. PLOS ONE 8, e55400 (2013)

38. Lau, D. H. et al. Epicardial border zone overexpression of skeletal muscle sodium channel SkM1 normalizes activation, preserves conduction, and suppresses ventricular arrhythmia: an in silico, in vivo, in vitro study. Circulation 119, 19-27 (2009).

39. Boink, G. J. et al. HCN2/SkM1 gene transfer into canine left bundle branch induces stable, autonomically responsive biological pacing at physiological heart rates. J. Am. Coll. Cardiol. 61, 1192-1201 (2013).

40. Subramanyam, P. et al. Manipulating L-type calcium channels in cardiomyocytes using split-intein protein transsplicing. Proc. Natl Acad. Sci. USA 110, 15461-15466 (2013).

41. Rog-Zielinska, E. A., Norris, R. A., Kohl, P. \& Markwald, R. the living scar--cardiac fibroblasts and the injured heart. Trends Mol. Med. 22, 99-114 (2016).

42. Chong, J. J. et al. Human embryonic-stem-cell-derived cardiomyocytes regenerate non-human primate hearts. Nature 510, 273-277 (2014)

43. Liao, S. Y. et al. Proarrhythmic risk of embryonic stem cell-derived cardiomyocyte transplantation in infarcted myocardium. Heart Rhythm 7, 1852-1859 (2010).

44. Tang, L. et al. Structural basis for $\mathrm{Ca} 2+$ selectivity of a voltage-gated calcium channel. Nature 505, 56-61 (2014).

45. Yue, L., Navarro, B., Ren, D., Ramos, A. \& Clapham, D. E. The cation selectivity filter of the bacterial sodium channel, NaChBac. J. Gen. Physiol. 120, 845-853 (2002).

46. Corry, B., Lee, S. \& Ahern, C. A. Pharmacological insights and quirks of bacterial sodium channels. Handb. Exp. Pharmacol. 221, 251-267 (2014).

47. Scheuer, T. Bacterial sodium channels: models for eukaryotic sodium and calcium channels. Handb. Exp. Pharmacol. 221, 269-291 (2014).

48. Blanchet, J., Pilote, S. \& Chahine, M. Acidic residues on the voltage-sensor domain determine the activation of the NaChBac sodium channel. Biophys. J. 92, 3513-3523 (2007).

49. Shaya, D. et al. Structure of a prokaryotic sodium channel pore reveals essential gating elements and an outer ion binding site common to eukaryotic channels. J. Mol. Biol. 426, 467-483 (2014).

50. Arrigoni, C. et al. Unfolding of a temperature-sensitive domain controls voltage-gated channel activation. Cell 164, 922-936 (2016).

51. Deisseroth, K. Optogenetics: 10 years of microbial opsins in neuroscience. Nat. Neurosci. 18, 1213-1225 (2015).

52. Cox, D. B., Platt, R. J. \& Zhang, F. Therapeutic genome editing: prospects and challenges. Nat. Med. 21, 121-131 (2015)

53. Cho, H. C. \& Marban, E. Biological therapies for cardiac arrhythmias: can genes and cells replace drugs and devices? Circ. Res. 106, 674-685 (2010).

54. Hu, T., Fu, Q., Chen, P., Zhang, K. \& Guo, D. Generation of a stable mammalian cell line for simultaneous expression of multiple genes by using $2 \mathrm{~A}$ peptide-based lentiviral vector. Biotechnol. Lett. 31, 353-359 (2009).

55. Klinger, R. \& Bursac, N. Cardiac cell therapy in vitro: reproducible assays for comparing the efficacy of different donor cells. IEEE Eng. Med. Biol. Mag. 27, 72-80 (2008).
56. Badie, N., Scull, J. A., Klinger, R. Y., Krol, A. \& Bursac, N. Conduction block in micropatterned cardiomyocyte cultures replicating the structure of ventricular cross-sections. Cardiovasc. Res. 93, 263-271 (2012).

57. Abbaci, M. et al. Gap junctional intercellular communication capacity by gapFRAP technique: a comparative study. Biotechnol. J. 2, 50-61 (2007).

58. McSpadden, L. C., Kirkton, R. D. \& Bursac, N. Electrotonic loading of anisotropic cardiac monolayers by unexcitable cells depends on connexin type and expression level. Am. J. Physiol. Cell. Physiol. 297, C339-C351 (2009).

59. Badie, N. \& Bursac, N. Novel micropatterned cardiac cell cultures with realistic ventricular microstructure. Biophys. J. 96, 3873-3885 (2009).

60. Bursac, N., Aguel, F. \& Tung, L. Multiarm spirals in a two-dimensional cardiac substrate. Proc. Natl Acad. Sci. USA 101, 15530-15534 (2004).

61. Protas, L. et al. Expression of skeletal but not cardiac $\mathrm{Na}+$ channel isoform preserves normal conduction in a depolarized cardiac syncytium. Cardiovasc. Res. 81, 528-535 (2009).

62. Pitts, K. R. \& Toombs, C. F. Coverslip hypoxia: a novel method for studying cardiac myocyte hypoxia and ischemia in vitro. Am. J. Physiol. Heart Circ. Physiol. 287, H1801-H1812 (2004).

63. Hodgkin, A. L. \& Huxley, A. F. Propagation of electrical signals along giant nerve fibers. Proc. R. Soc. Lond. B 140, 177-183 (1952).

64. ten Tusscher, K. H., Noble, D., Noble, P. J. \& Panfilov, A. V. A model for human ventricular tissue. Am. J. Physiol. Heart Circ. Physiol. 286, H1573-H1589 (2004).

65. Nygren, A. et al. Mathematical model of an adult human atrial cell: the role of $\mathrm{K}+$ currents in repolarization. Circ. Res. 82, 63-81 (1998).

66. Hodgkin, A. L. \& Rushton, W. A. The electrical constants of a crustacean nerve fibre. Proc. R. Soc. Med. 134, 444-479 (1946).

67. Rall, W. in Comprehensive Physiology (John Wiley \& Sons, Inc., 2011).

68. Shaw, R. M. \& Rudy, Y. Ionic mechanisms of propagation in cardiac tissue. Roles of the sodium and L-type calcium currents during reduced excitability and decreased gap junction coupling. Circ. Res. 81, 727-741 (1997).

\section{Acknowledgements}

We thank A. Krol and C. Jackman for assistance with neonatal rat cardiomyocyte culture, N. Gillum and K. Kanagasegar for assistance with the development of computational model of $\mathrm{BacNa}_{\mathrm{v}}$, and L. Li for construction of lentiviral libraries. We further thank V. Bennett and L. You for critical reading of the manuscript. This work was supported by American Heart Association Predoctoral Fellowship PRE 16790012 to H.X.N. and National Institutes of Health grants HL104326, HL132389, HL126524 and HL126193.

\section{Author contributions}

H.X.N. and N.B. designed the project. H.X.N. and R.D.K. performed experiments. H.X.N. and N.B. analysed data and co-wrote the manuscript.

\section{Additional information}

Supplementary Information accompanies this paper at http://www.nature.com/ naturecommunications

Competing financial interests: The authors declare no competing financial interests.

Reprints and permission information is available online at http://npg.nature.com/ reprintsandpermissions/

How to cite this article: Nguyen, H. X. et al. Engineering prokaryotic channels for control of mammalian tissue excitability. Nat. Commun. 7, 13132 doi: $10.1038 /$ ncomms13132 (2016)

This work is licensed under a Creative Commons Attribution 4.0 International License. The images or other third party material in this article are included in the article's Creative Commons license, unless indicated otherwise in the credit line; if the material is not included under the Creative Commons license, users will need to obtain permission from the license holder to reproduce the material. To view a copy of this license, visit http://creativecommons.org/licenses/by/4.0/ 